\title{
Singular Poisson Reduction of Cotangent Bundles
}

\author{
Simon HOCHGERNER and Armin RAINER \\ Institut für Mathematik \\ Universität Wien \\ Nordbergstrasse 15 \\ A-1090 Vienna - Austria \\ simon.hochgerner@univie.ac.at armin.rainer@univie.ac.at
}

Received: January 23, 2006

Accepted: May 19, 2006

\begin{abstract}
We consider the Poisson reduced space $\left(T^{*} Q\right) / K$, where the action of the compact Lie group $K$ on the configuration manifold $Q$ is of single orbit type and is cotangent lifted to $T^{*} Q$. Realizing $\left(T^{*} Q\right) / K$ as a Weinstein space we determine the induced Poisson structure and its symplectic leaves. We thus extend the Weinstein construction for principal fiber bundles to the case of surjective Riemannian submersions $Q \rightarrow Q / K$ which are of single orbit type.
\end{abstract}

Key words: cotangent bundle reduction, singular reduction, Poisson structures.

2000 Mathematics Subject Classification: 53D17, 53D20.

\section{Introduction}

The present paper deals with Poisson reduction of a cotangent bundle $T^{*} Q$ with respect to a Hamiltonian action by a compact Lie group $K$ that comes as the cotangent lifted action from the configuration manifold $Q$. We assume that $Q$ is Riemannian and $K$ acts on $Q$ by isometries. Further, we suppose that $Q$ is of single isotropy type, i.e., $Q=Q_{(H)}$ for some subgroup $H$ of $K$. The cotangent bundle $T^{*} Q$ is equipped with its canonical symplectic form, and we have a standard momentum map $\mu: T^{*} Q \rightarrow \mathfrak{k}^{*}$. Then the orbit space $Q / K$ is a smooth manifold. In the presence of a single non-trivial isotropy on the configuration space $Q$ one gets a non-trivial isotropy lattice on $T^{*} Q$.

This work is supported by Fonds zur Förderung der wissenschaftlichen Forschung, Projekt P 17108-N04. 
In Hochgerner [14] stratified symplectic reduction of $T^{*} Q$ was studied under these assumptions. In particular, the following result was proved, following an approach that is generally called Weinstein construction: Let $\mathcal{O}$ be a coadjoint orbit lying in the image of the standard momentum map $\mu$. Then each smooth symplectic stratum $\left(T^{*} Q / /{ }_{\mathcal{O}} K\right)_{(L)}$ of the reduced space can be globally realized as

$$
\left(\mathcal{W} / /{ }_{\mathcal{O}} K\right)_{(L)}=T^{*}(Q / K) \times{ }_{Q / K}\left(\bigsqcup_{q \in Q} \mathcal{O} \cap \operatorname{Ann} \mathfrak{k}_{q}\right)_{(L)} / K
$$

where

$$
\mathcal{W}:=\left(Q \times_{Q / K} T^{*}(Q / K)\right) \times_{Q} \bigsqcup_{q \in Q} \operatorname{Ann}_{q} \cong T^{*} Q
$$

as symplectic manifolds with a Hamiltonian $K$-action. Moreover, the reduced symplectic structure in terms intrinsic to this realization was computed. These results were applied to Calogero-Moser systems with spin associated to polar representations of compact Lie groups.

In the above setting the reduced space $T^{*} Q / K$ is a (singular) Poisson space in a natural way. The underlying theorem due to Ortega and Ratiu [19] is presented in 2.2. The goal of this paper is to determine the reduced Poisson bracket on $T^{*} Q / K$.

In section 2 we give the necessary background from singular geometry.

Section 3 presents the basic setting and the prerequisites of the paper, in particular, recalling from Hochgerner [14] the construction of the Weinstein space $\mathcal{W}$ and the intrinsic symplectic structure on it.

Finally, in section 4 we compute the reduced gauged Poisson bracket on the Weinstein realization $\mathcal{W} / K$ of $T^{*} Q / K$. This is done by determining the Poisson bracket on $\mathcal{W}$ via the symplectic structure intrinsic to this space and then using the identification $C^{\infty}(\mathcal{W} / K) \cong C^{\infty}(\mathcal{W})^{K}$. In particular, it will be important to introduce a suitably chosen linear connection $\widetilde{A}$ on the bundle

$$
\mathcal{W} \longrightarrow Q \times_{Q / K} T^{*}(Q / K)
$$

The formula for the reduced Poisson bracket on $\mathcal{W} / K$ will involve the canonical Poisson structure on $T^{*}(Q / K)$, the pairing of a curvature term associated to the mechanical connection $A$ on $Q \rightarrow Q / K$ (see section 3) with the appropriate point of $\bigsqcup_{q \in Q}$ Ann $\mathfrak{k}_{q}$, and the Lie bracket of the fiber derivatives on $\bigsqcup_{q \in Q}$ Ann $\mathfrak{k}_{q}$.

Note that this result generalizes the formula for the reduced Poisson bracket on $T^{*} Q / K$ presented in Zaalani [34] and in Perlmutter and Ratiu [23] for the case that $K$ acts on $Q$ freely.

In section 5 we compute the symplectic leaves of the reduced space $\mathcal{W} / K$, and in section 6 we give examples of our constructions. 


\section{Conventions}

Let $K$ be a Lie group acting on a manifold $M$. In fact, we will only be concerned with the case where $K$ is compact, $M$ is Riemannian, and $K$ acts on $M$ through isometries, i.e., $M$ is a Riemannian $K$-space. The action will be written as $l: K \times M \rightarrow M$, $(k, x) \mapsto l(k, x)=l_{k}(x)=l^{x}(k)=k . x$. Sometimes the action will be lifted to the tangent bundle $T M$. That is, we will consider $h \cdot(x, v):=(h \cdot x, h \cdot v):=$ $T l_{h} \cdot(x, v)=\left(l_{h}(x), T_{x} l_{h} \cdot v\right)$ where $(x, v) \in T M$. As the action is a transformation by a diffeomorphism it may also be lifted to the cotangent bundle. This is the cotangent lifted action which is defined by $h \cdot(x, p):=(h \cdot x, h \cdot p):=T^{*} l_{h} \cdot(x, p)=\left(h \cdot x, T_{h \cdot x}^{*} l_{h^{-1}} \cdot p\right)$ where $(x, p) \in T^{*} M$.

The fundamental vector field is going to play repeatedly an important role. It is defined by

$$
\zeta_{X}(x):=\left.\frac{\partial}{\partial t}\right|_{0} l(\exp (+t X), x)=T_{e} l^{x}(X)
$$

where $X \in \mathfrak{k}$. The fundamental vector field mapping $\mathfrak{k} \rightarrow \mathfrak{X}(M), X \mapsto \zeta_{X}$ is a Lie algebra anti-homomorphism. By definition the flow of $\zeta_{X}$ is given by $l_{\exp (t X)}$.

If the action by $K$ on $M$ is proper, in the sense that $K \times M \rightarrow M \times M,(k, x) \mapsto$ $(x, l(k, x))$ is a proper mapping, then we have the Slice and Tube Theorem at our disposal. Properness is automatic for $K$ compact. An exposition of these facts can be found in Palais and Terng [22] or Ortega and Ratiu [20], for example.

Let $H$ be a subgroup of $K$. A point $x \in M$ is said to be of isotropy or orbit type $H$ if its isotropy group $K_{x}=\{k \in K: k . x=x\}$ is conjugate to $H$ within $K$ for which we shall write $K_{x} \sim H$. The family of subgroups of $K$ conjugate to $H$ within $K$ is denoted by $(H)$ and called the conjugacy class of $H$. We will deal with the isotropy or orbit type submanifold $M_{(H)}:=\left\{x \in M: K_{x} \sim H\right\}$ of type $H$, the set $M_{H}:=\left\{x \in M: K_{x}=H\right\}$ of points that have symmetry type $H$, and the set $\operatorname{Fix}(H):=M^{H}:=\left\{x \in M: H \subseteq K_{x}\right\}$ of points that are fixed by $H$. Then $M_{(H)}$ is a submanifold of $M, \operatorname{Fix}(H)$ is a totally geodesic submanifold of $M$, and $M_{H}$ is an open submanifold of $\operatorname{Fix}(H)$.

If $(M, \omega)$ is a symplectic manifold we define the associated Poisson bracket and Hamiltonian vector field by

$$
\{f, g\}=\omega\left(\nabla_{f}^{\omega}, \nabla_{g}^{\omega}\right)=-\nabla_{f}^{\omega}(g)
$$

where $f, g \in C^{\infty}(M)$. This choice of sign is compatible with that in [20]. It is, however, not universal.

\section{Singular geometry}

\subsection{Singular spaces and smooth structures}

First we introduce the Whitney condition $(b)$ which will be necessary in the definition of Whitney stratified spaces — see Definition 2.8. We follow the approach of 
Mather [17].

Definition 2.1 (Whitney condition $(b)$ in $\mathbb{R}^{n}$ ). Let $X, Y$ be disjoint sub-manifolds of $\mathbb{R}^{n}$ with $\operatorname{dim} X=r$. The pair $(X, Y)$ is said to satisfy condition $(b)$ at $y \in Y$ if the following is true. Consider sequences $\left(x_{i}\right)_{i},\left(y_{i}\right)_{i}$ in $X, Y$, respectively, such that $x_{i} \rightarrow y$ and $y_{i} \rightarrow y$. Assume that $T_{x_{i}} X$ converges to some $r$-plane $\tau \subseteq T_{y} \mathbb{R}^{n}=\mathbb{R}^{n}$, and that the lines spanned by the vectors $y_{i}-x_{i}$ converge - in $\mathbb{R} P^{n-1}$ — to some line $l \subseteq \mathbb{R}^{n}=T_{y} \mathbb{R}^{n}$. Then $l \subseteq \tau$. The pair $(X, Y)$ satisfies condition (b) if it does so at every $y \in Y$.

Obviously condition $(b)$ behaves well under diffeomorphisms in the following sense: for $i=1,2$ consider pairs $\left(X_{i}, Y_{i}\right)$ in $\mathbb{R}^{n}$, points $y_{i} \in Y_{i}$, open neighborhoods $U_{i} \subseteq \mathbb{R}^{n}$ of $y_{i}$, and a diffeomorphism $\phi: U_{1} \rightarrow U_{2}$ sending $y_{1}$ to $y_{2}$ and satisfying $\phi\left(U_{1} \cap X_{1}\right)=$ $U_{2} \cap X_{2}$ as well as $\phi\left(U_{1} \cap Y_{1}\right)=U_{2} \cap Y_{2}$. Thus it makes sense to formulate this condition for manifolds.

Definition 2.2 (Whitney condition $(b)$ ). Let $M$ be a manifold and $X, Y$ disjoint sub-manifolds. Now $(X, Y)$ is said to satisfy condition $(b)$ if the following holds for all $y \in Y$. Let $(U, \phi)$ be a chart around $y$. Then the pair $(\phi(X \cap U), \phi(Y \cap U))$ satisfies condition $(b)$ at $\phi(y)$.

By the above this definition is independent of the chosen chart in the formulation.

Example 2.3. Consider $M=\mathbb{C}^{3}=\{(x, y, z)\}$ with $Y$ the $z$-axis, and $X=\{(x, y, z)$ : $\left.y^{2}+x^{3}-z^{2} x^{2}=0\right\} \backslash Y$. Then the pair $(X, Y)$ satisfies condition $(b)$ at all points in $Y$ except at $y=0$. Notice that we can refine the decomposition of $M$ as $(X, Y \backslash\{0\},\{0\})$. Now all pairs in this finer decomposition satisfy condition $(b)$.

Let $X$ be a para-compact and second countable topological Hausdorff space, and let $(I, \leq)$ be a partially ordered set.

Definition 2.4 (Decomposed space). An $I$-decomposition of $X$ is a locally finite partition of $X$ into smooth manifolds $S_{i}, i \in I$ which are disjoint (but may consist of finitely many connected components with differing dimension), and satisfy:

(i) Each $S_{i}$ is locally closed in $X$;

(ii) $X=\bigcup_{i \in I} S_{i}$;

(iii) $S_{j} \cap \overline{S_{i}} \neq \emptyset \Longleftrightarrow S_{j} \subseteq \overline{S_{i}} \Longleftrightarrow j \leq i$.

The third condition is called condition of the frontier. The manifolds $S_{i}$ are called strata or pieces. In the case that $j<i$ one often writes $S_{j}<S_{i}$ and calls $S_{j}$ incident to $S_{i}$ or says $S_{j}$ is a boundary piece of $S_{i}$.

We define the dimension of a manifold consisting of finitely many connected components to be the maximum of the dimensions of the manifold's components. 
The dimension of the decomposed space $X$ is defined as

$$
\operatorname{dim} X:=\sup _{i \in I} \operatorname{dim} S_{i}
$$

and we will only be concerned with spaces where this supremum is attained.

The depth of the stratum $S_{i}$ of the decomposed space $X$ is defined as

$$
\begin{array}{r}
\operatorname{depth} S_{i}:=\sup \left\{l \in \mathbb{N}: \text { there are strata } S_{i_{0}}=S_{i}, S_{i_{1}}, \ldots, S_{i_{l}}\right. \\
\text { such that } \left.S_{i_{0}}<\cdots<S_{i_{l}}\right\},
\end{array}
$$

Notice that depth $S_{i}$ is always finite; indeed, otherwise there would be an infinite family $\left(S_{j}\right)_{j \in J}$ with $S_{j}>S_{i}$ thus making any neighborhood of any point in $S_{i}$ meet all of the $S_{j}$ which contradicts local finiteness of the decomposition. The depth of $X$ is

$$
\operatorname{depth} X:=\sup \left\{\operatorname{depth} S_{i}: i \in I\right\} .
$$

Thus, if $X$ consists of just one stratum, then depth $X=0$. From the frontier condition we have that $\operatorname{depth} S_{i} \leq \operatorname{dim} X-\operatorname{dim} S_{i}$, and also $\operatorname{depth} X \leq \operatorname{dim} X$.

A simple example for a decomposed space is a manifold with boundary with big stratum the interior and small stratum the boundary. Also manifolds with corners are decomposed spaces in the obvious way. Likewise the cone $C M:=(M \times[0, \infty)) /$ $(M \times\{0\})$ over a manifold $M$ is a decomposed space, the partition being that into cusp and open cylinder $M \times(0, \infty)$.

The following definition of singular charts and smooth structures on singular spaces is due to Pflaum [25, section 2].

Definition 2.5 (Singular charts). Let $X=\bigcup_{i \in I} S_{i}$ be a decomposed space. A singular chart $(U, \psi)$ with patch $U$ an open subset of $X$ is to satisfy the following.

(i) $\psi(U)$ is locally closed in $\mathbb{R}^{n}$;

(ii) $\psi: U \rightarrow \psi(U)$ is a homeomorphism;

(iii) For every stratum $S_{i}$ that meets $U$ the restriction $\psi \mid S_{i} \cap U: S_{i} \cap U \rightarrow \psi\left(S_{i} \cap U\right)$ is a diffeomorphism onto a smooth sub-manifold of $\mathbb{R}^{n}$.

Two singular charts $\psi: U \rightarrow \mathbb{R}^{n}$ and $\phi: V \rightarrow \mathbb{R}^{m}$ are called compatible at $x \in U \cap V$ if there is an open neighborhood $W$ of $x$ in $U \cap V$, a number $N \geq \max \{n, m\}$, and a diffeomorphism $f: W_{1} \rightarrow W_{2}$ between open subsets of $\mathbb{R}^{N}$ such that:

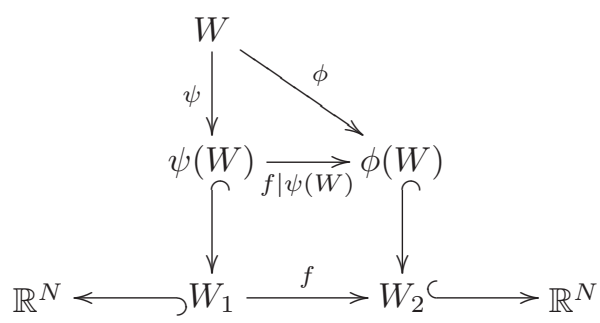


It follows that $f \mid \psi(W): \psi(W) \rightarrow \phi(W)$ is a homeomorphism. Further, for all strata $S$ that meet $W$ the restriction $f \mid \psi(W \cap S): \psi(W \cap S) \rightarrow \phi(W \cap S)$ is a diffeomorphism of sub-manifolds of $\mathbb{R}^{N}$. The charts $(U, \psi)$ and $(V, \phi)$ are called compatible if they are so at every point of the intersection $U \cap V$. It is straightforward to check that compatibility of charts defines an equivalence relation.

A family of compatible singular charts on $X$ such that the union of patches covers all of $X$ is called a singular atlas. Two singular atlases are said to be compatible if all charts of the first are compatible with all charts of the second. Again it is clear that compatibility of atlases forms an equivalence relation.

Let $\mathfrak{A}$ be a singular atlas on $X$. Then we can consider the family of all singular charts that belong to some atlas compatible with $\mathfrak{A}$ to obtain a maximal atlas $\mathfrak{A}_{\max }$.

Definition 2.6 (Smooth structure). Let $X=\bigcup_{i \in I} S_{i}$ be a decomposed space. A maximal atlas $\mathfrak{A}$ on $X$ is called a smooth structure on the singular space $X$. A continuous function $f: X \rightarrow \mathbb{R}$ is said to be smooth if the following holds. For all charts $\psi: U \rightarrow \mathbb{R}^{n}$ of the atlas $\mathfrak{A}$ there is a smooth function $F: \mathbb{R}^{n} \rightarrow \mathbb{R}$ such that $f \mid U=F \circ \psi$. The set of all smooth functions on $X$ is denoted by $C^{\infty}(X)$.

A continuous map $f: X \rightarrow Y$ between decomposed spaces with smooth structures is called smooth if $f^{*} C^{\infty}(Y) \subseteq C^{\infty}(X)$. An isomorphism $F: X \rightarrow Y$ between decomposed spaces is a homeomorphism that is smooth in both directions and maps strata of $X$ diffeomorphically onto strata of $Y$.

The smooth structure thus defined on decomposed spaces is in no way intrinsic but is a structure that is additionally defined to do analysis on decomposed spaces. Also a smooth map $f: X \rightarrow Y$ between decomposed spaces need not at all be strata preserving.

Definition 2.7 (Cone space). A decomposed space $X=\bigcup_{i \in I} S_{i}$ is called a cone space if the following is true. Let $x_{0} \in X$ arbitrary and $S$ the stratum passing through $x_{0}$. Then there is an open neighborhood $U$ of $x_{0}$ in $X$, there is a decomposed space $L$ with global chart $\psi: L \rightarrow S^{l-1} \subseteq \mathbb{R}^{l}$, and furthermore there is an isomorphism of decomposed spaces

$$
F: U \rightarrow(U \cap S) \times C L
$$

such that $F(x)=(x, c)$ for all $x \in U \cap S$. Here $C L=(L \times[0, \infty)) /(L \times\{0\})$ is decomposed into the cusp $c$ on the one hand, while the other pieces are of the form stratum of $L$ times $(0, \infty)$. Thus we can take $\Psi: C L \rightarrow \mathbb{R}^{l},[(z, t)] \mapsto t \psi(z)$ as a global chart on $C L$ thereby defining a smooth structure on $C L$ whence also on the product $(U \cap S) \times C L$.

The space $L$ is called a link, and the chart $F$ is referred to as a cone chart or also a link chart. Of course, the link $L$ depends on the chosen point $x_{0} \in X$.

An example for a cone space is the quadrant $Q:=\left\{(x, y) \in \mathbb{R}^{2}: x \geq 0\right.$ and $\left.y \geq 0\right\}$. A typical neighborhood of $0 \in Q$ is of the form $\{(x, y): 0 \leq x<r$ and $0 \leq y<r\}$. 
The link with respect to the point 0 then is the $\operatorname{arc} L:=\left\{(\cos \varphi, \sin \varphi): 0 \leq \varphi \leq \frac{\pi}{2}\right\}$. More generally manifolds with corners carry the structure of cone spaces.

Definition 2.8 (Stratified spaces). Let $X \subseteq \mathbb{R}^{m}$ be a subset and assume that $X$ is a decomposed space, i.e. $X=\bigcup_{i \in I} S_{i}$, and that the strata $S_{i}$ be sub-manifolds of $\mathbb{R}^{m}$. The $I$-decomposed space $X$ is said to be (Whitney) stratified if all pairs $\left(S_{i}, S_{j}\right)$ with $i>j$ satisfy condition $(b)-$ see Definition 2.2. For sake of convenience we will simply say stratified instead of Whitney stratified.

Theorem 2.9. Let $X \subseteq \mathbb{R}^{m}$ be a subset of a Euclidean space and assume that $X=$ $\bigcup_{i \in I} S_{i}$ is decomposed. Then $X$ is stratified if and only if $X$ is a cone space.

Proof. It is proved in Pflaum [26] that every (Whitney) stratified space is also a cone space.

An outline of the converse direction is given in Sjamaar and Lerman [29, section 6], and also in Goresky and MacPherson [12, section 1.4]. This argument makes use of Mather's control theory as introduced in Mather [17] as well as Thom's First Isotopy Lemma.

The above theorem depends crucially on the fact that the decomposed space $X$ can be regarded as a subspace of some Euclidean space. As this assumption will always be satisfied in the present context we will take the words cone space and stratified space to be synonymous. In fact, Sjamaar and Lerman [29] take cone space to be the definition of stratified space.

Example 2.10. As an example consider a compact Lie group $K$ acting by isometries on a smooth Riemannian manifold $M$. We are concerned with the orbit projection $\pi: M \rightarrow M / K$ and endow the orbit space with the final topology with respect to the projection map. For basics on compact transformation groups see Bredon [7], Palais and Terng [22], or Hochgerner [14, section 7]. Fix a point $x_{0} \in M$ with isotropy group $K_{x_{0}}=H$. The slice representation is then the action by $H$ on $\operatorname{Nor}_{x_{0}}\left(K \cdot x_{0}\right)=$ $T_{x_{0}}\left(K \cdot x_{0}\right)^{\perp}$. By the Tube Theorem there is a $K$-invariant open neighborhood $U$ of the orbit $K \cdot x_{0}$ such that $K \times_{H} V \cong U$ as smooth $K$-spaces where $V$ is an $H$-invariant open neighborhood of 0 in $\operatorname{Nor}_{x_{0}}\left(K \cdot x_{0}\right)$.

Now let $p=\left(p_{1}, \ldots, p_{k}\right)$ be a Hilbert basis for the algebra $\operatorname{Poly}(V)^{H}$ of $H$-invariant polynomials on $V$. That is, $p_{1}, \ldots, p_{k}$ is a finite system of generators for $\operatorname{Poly}(V)^{H}$. The Theorem of Schwarz [28, Theorem 1] now says that $p^{*}: C^{\infty}\left(\mathbb{R}^{k}\right) \rightarrow C^{\infty}(V)^{H}$ is surjective. Moreover, the induced mapping $q: V / H \rightarrow \mathbb{R}^{k}$ is continuous, injective, and proper. See also Michor [18].

Consider the isotropy type sub-manifolds $M_{(H)}$ which is the manifold of all points of $M$ whose isotropy subgroup is conjugate to $H$ within $K$. These give a $K$-invariant decomposition of $M$ as $M=\bigcup_{(H)} M_{(H)}$ where $(H)$ runs through the isotropy lattice of the $K$-action on $M$. We thus get a decomposition of the orbit space

$$
M / K=\bigcup_{(H)} M_{(H)} / K
$$


where again $(H)$ runs through the isotropy lattice of the $K$-action on $M$. It is wellknown (e.g., Palais and Terng [22]) that this decomposition renders $M / K$ a decomposed space.

Now a theorem of Pflaum [25, Theorem 5.9] says that the induced mapping $\psi$ : $U / K \rightarrow \mathbb{R}^{k}$ as defined in the diagram

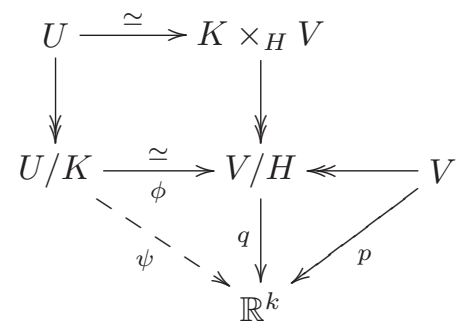

is a typical singular chart around the point $K . x_{0}$ in the orbit space. Furthermore, the smooth functions with respect to this smooth structure are $C^{\infty}(M / K)=C^{\infty}(M)^{K}$, i.e., none other than the $K$-invariant smooth functions on $M$ : indeed, by Schwarz's Theorem we have

$$
\psi^{*} C^{\infty}\left(\mathbb{R}^{k}\right)=\phi^{*} q^{*} C^{\infty}\left(\mathbb{R}^{k}\right)=\phi^{*} C^{\infty}(V)^{H}=C^{\infty}(U)^{K}
$$

whence $C^{\infty}(U)^{K}=C^{\infty}(U / K)$. Finally, the decomposition of $M / K$ by orbit types turns the orbit space into a stratified space with smooth structure.

Note that Bierstone [5] showed that the semi-analytic stratification of the orbit space $p(V)$ of a linear $K$-space $V$ coincides with its stratification by components of sub-manifolds of given isotropy type. Here $K$ is a compact Lie group and $p$ is a Hilbert basis as above. Further, the semi-analytic stratification of $p(V)$ satisfies Whitney's condition $(b)$.

\subsection{Singular Poisson reduction}

Let $K$ be a Lie group acting properly on a smooth manifold $M$. We equip the orbit space $M / K$ with the quotient topology with respect to the canonical projection $\pi: M \rightarrow M / K$. The set of smooth functions on $M / K$ is defined by the requirement that $\pi$ is a smooth map, i.e.,

$$
C^{\infty}(M / K):=\left\{f \in C^{0}(M / K): f \circ \pi \in C^{\infty}(M)\right\} .
$$

Theorem (Singular Poisson reduction). Let $(M,\{\cdot, \cdot\})$ be a Poisson manifold, $K$ a Lie group, and let $l: K \times M \rightarrow M$ be a smooth proper Poisson action, i.e., $l_{k}^{*}\{f, g\}=\left\{l_{k}^{*} f, l_{k}^{*} g\right\}$ for $f, g \in C^{\infty}(M)$ and $k \in K$. Then we have:

(i) The pair $\left(C^{\infty}(M / K),\{\cdot, \cdot\}^{M / K}\right)$ is a Poisson algebra, where the Poisson bracket $\{\cdot, \cdot\}^{M / K}$ is characterized by $\{f, g\}^{M / K} \circ \pi=\{f \circ \pi, g \circ \pi\}$, for any $f, g \in$ $C^{\infty}(M / K)$, and $\pi: M \rightarrow M / K$ denotes the canonical smooth projection. 
(ii) Let $h \in C^{\infty}(M)^{K}$ be a $K$-invariant function on $M$. The flow $\mathrm{Fl}_{t}$ of the Hamiltonian vector field associated to $h$ commutes with the $K$-action, so it induces a flow $\mathrm{Fl}_{t}^{M / K}$ on $M / K$ which is Poisson and is characterized by the identity $\pi \circ \mathrm{Fl}_{t}=\mathrm{Fl}_{t}^{M / K} \circ \pi$.

(iii) The flow $\mathrm{Fl}_{t}^{M / K}$ is the unique Hamiltonian flow defined by the function $H \in$ $C^{\infty}(M / K)$ which is given by $H \circ \pi=h$.

Proof. This is due to Ortega and Ratiu [19].

If, in particular, $K$ is a compact Lie group acting by isometries on a smooth Riemannian manifold $M$, then by the Tube Theorem [22] and Schwarz's Theorem [28] we may identify $C^{\infty}(M / K)$ with $C^{\infty}(M)^{K}$, the set of $K$-invariant smooth functions on $M$. See Example 2.10 for details.

Definition 2.11 (Poisson stratified space). Let $X$ be a stratified space endowed with a smooth structure in the sense of Subsection 2.1. Then $X$ is said to be a singular Poisson space if there is a Poisson bracket

$$
\{\cdot, \cdot\}: C^{\infty}(X) \times C^{\infty}(X) \longrightarrow C^{\infty}(X)
$$

on the algebra of smooth functions determined by the smooth structure such that the inclusion of each stratum $S \hookrightarrow X$ is a Poisson morphism. In particular, the strata $S$ are Poisson manifolds in the usual sense.

An alternative definition of a singular Poisson space in terms of a stratified Poisson bivector is given by Pflaum [25].

Proposition 2.12 (Reduced Poisson structure). Let $(M,\{\cdot, \cdot\})$ be a Poisson manifold, $K$ a compact Lie group, and let $K$ act on $M$ by Poisson morphisms. Then $\left(M / K,\{\cdot, \cdot\}^{M / K}\right)$ is a singular Poisson space.

Proof. By Example 2.10 the algebra $C^{\infty}(M / K)$ is indeed determined by a smooth structure on $M / K$. Thus it only remains to check that the inclusion of each stratum $M_{(H)} / K \hookrightarrow M / K$ is a Poisson morphism. This is, however, obvious.

\subsection{Singular fiber bundles}

Definition 2.13 (Singular fiber bundles). Let $F$ and $P$ be stratified spaces (Definition 2.8) with smooth structure (Definition 2.6) and $M$ be a smooth manifold. We say that the topological fiber bundle

$$
F^{\longrightarrow} P \stackrel{\pi}{\longrightarrow} M
$$

is a singular fiber bundle if for each trivializing patch $U \subseteq M$ the homeomorphism

$$
P \mid U \cong U \times F
$$

is an isomorphism of stratified spaces. 
There are two reasons for defining singular fiber bundles in this way. Firstly, it is the kind of structure encountered in Hochgerner [14, Theorem 5.5], and secondly by Mather's control theory [17] these bundles possess many features similar to ordinary smooth fiber bundles. Some of these aspects are presented in the subsections below.

Note that if $M$ is a Riemannian manifold which is acted upon by a compact Lie group $K$ through isometries then the orbit projection mapping $M \rightarrow M / K$ is, in general, not a singular fiber bundle according to this definition. Indeed, the fiber type of $M \rightarrow M / K$ needs not to be locally constant.

Lemma 2.14. Let $\pi: P \rightarrow M$ be a singular fiber bundle with typical fiber $F$. Let $S$ be a stratum of $P$. Then $\pi \mid S: S \rightarrow M$ is a smooth fiber bundle.

Proof. Indeed, locally the stratum $S$ is diffeomorphic to $U \times S_{F}$ where $S_{F}$ is a stratum of $F$ and $U$ is a trivializing neighborhood in $M$.

Definition 2.15 (Singular symplectic fiber bundles). Let $F$ and $P$ be stratified symplectic spaces (i.e., singular Poisson spaces with smooth symplectic strata such that the inclusion mappings are Poisson morphisms) with smooth structure and $M$ be a smooth symplectic manifold. We say that the singular fiber bundle

$$
F^{\complement} \longrightarrow P \stackrel{\pi}{\longrightarrow} M
$$

is a singular symplectic fiber bundle if for each trivializing patch $U \subseteq M$ the homeomorphism

$$
P \mid U \cong U \times F
$$

is an isomorphism of stratified symplectic spaces with respect to the inherited symplectic structures. It follows, in particular, that $\pi$ is a Poisson morphism.

\subsection{Control data}

The theory of control data is due to Mather [17], and we follow in our presentation of the subject that of [17]. Let $N$ be a smooth manifold, and $X \subseteq N$ a stratified subset endowed with the relative topology with strata $S_{i}$ where $i \in I$ as in Subsection 2.1.

A tubular neighborhood of a stratum $S_{i}$ in $X$ is a closed neighborhood of $S_{i}$ in $N$ which is diffeomorphic to an inner product bundle $\pi_{i}: E_{i} \rightarrow S_{i}$. Via the inner product we can measure the vertical distance of a point in $E_{i}$ to $S_{i}$ and call this the tubular neighborhood function $\rho_{i}: E_{i} \rightarrow \mathbb{R}$. Clearly, $\rho_{i}(x)=0$ if and only if $x \in S_{i}$. We can also think of the tubular neighborhood as being retracted onto $S_{i}$ via the projection $\pi_{i}$. Control data associated to the stratification $\left\{S_{i}: i \in I\right\}$ of $X$ is a system of tubular neighborhoods $\pi_{i}: E_{i} \rightarrow S_{i}$ satisfying the following commutation relations:

$$
\left(\pi_{j} \circ \pi_{i}\right)(x)=\pi_{j}(x) \text { and }\left(\rho_{j} \circ \pi_{i}\right)(x)=\rho_{j}(x)
$$

whenever $j<i$ and both sides are defined. 
Proposition 2.16. Suppose there exist control data to the stratification $\left\{S_{i}: i \in I\right\}$ of $X$. If $M$ is another manifold, and $f: N \rightarrow M$ a smooth mapping such that $f \mid S_{i}: S_{i} \rightarrow M$ is a submersion for all $i \in I$ then the control data may be chosen so that $f \mid S_{i} \circ \pi_{S_{i}}=f$ for all $i \in I$.

Proof. See Mather [17, Proposition 7.1].

If $f: N \rightarrow M$ is as in Proposition 2.16 then $f$ is said to be a controlled submersion from $X$ to $M$.

By a stratified vector field $\eta$ on $X$ we mean a collection $\left\{\eta_{i}: i \in I\right\}$ where each $\eta_{i}$ is a smooth vector field on $S_{i}$. Assume we are given a system of control data associated to the stratification of $X$, and identify the tubular neighborhoods of the strata with the corresponding inner product bundles. Then the stratified vector field $\eta$ on $X$ is said to be a controlled vector field if the following conditions are met. For any stratum $S_{j}$ there is an open neighborhood $B_{j}$ of $S_{j}$ in the tubular neighborhood $E_{j}$ such that for any stratum $S_{i}$ with $i>j$ the conditions

$$
\mathcal{L}_{\eta_{i}}\left(\rho_{j} \mid B_{j} \cap S_{i}\right)=0 \quad \text { and } \quad T_{x}\left(\pi_{j} \mid B_{j} \cap S_{i}\right) \cdot \eta_{i}(x)=\eta_{j}\left(\pi_{j}(x)\right)
$$

are satisfied for all $x \in B_{j} \cap S_{i}$.

Let $J$ be an open neighborhood of $\{0\} \times X$ in $\mathbb{R} \times X$, and assume that $\alpha: J \rightarrow X$ is a local one-parameter group which is smooth in the sense of Definition 2.6. We say that $\alpha$ generates the stratified vector field $\eta$ if $J$ is maximal such that each stratum $S_{i}$ is invariant under $\alpha$ and $\left.\frac{\partial}{\partial t}\right|_{0} \alpha(t, x)=\eta_{i}(x)$ for all $x \in S_{i}$ and all $i \in I$.

Proposition 2.17. Assume $\eta$ is a controlled vector field on $X$. Then there is a unique smooth one-parameter group which generates $\eta$.

Proof. See Mather [17, Proposition 10.1].

Proposition 2.18. Assume $f: N \rightarrow M$ is a smooth map such that $f \mid X: X \rightarrow M$ is a controlled surjective submersion. Then the following are true.

- Let $\xi$ be a smooth vector field on $M$. Then there is a controlled vector field $\eta$ on $X$ such that $\eta_{i}$ and $\xi$ are $f \mid S_{i}$-related for all $i \in I$.

- Suppose further that $f \mid X: X \rightarrow M$ is a proper map. Then $f \mid X: X \rightarrow M$ is a singular fiber bundle.

Proof. See Mather [17, Proposition 9.1] for the first statement. Concerning the second assertion, [17, Proposition 11.1] states that under these assumptions the mapping $f \mid X: X \rightarrow M$ is locally topologically trivial, and it follows from [17, Corollary 10.3] that the trivializing homeomorphisms are, in fact, isomorphisms of stratified spaces. Thus $f \mid X: X \rightarrow M$ is a singular fiber bundle in the sense of Definition 2.13. 


\subsection{Pullback bundles}

Let $M$ and $Y$ be smooth manifolds, and let $\tau: Y \rightarrow M$ be a smooth mapping. Consider a singular fiber bundle $\pi: X \rightarrow M$ with typical fiber $F$ as in Definition 2.13. We consider further the topological pullback bundle of $X$ and $Y$ over $M$ with the following notation.

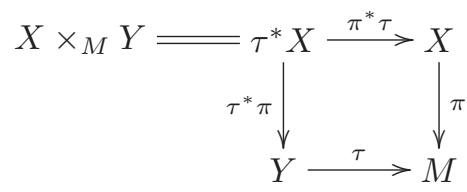

Now we can endow $X \times_{M} Y$ with the product stratification given by strata of the form $S \times{ }_{M} Y$ which is the smooth fibered product of a stratum $S$ of $X$ with $Y$ over $M$. Note that $S \times{ }_{M} Y$ is a well defined pull back bundle by Lemma 2.14. Moreover, $X \times_{M} Y$ inherits a smooth structure in the sense of Subsection 2.1 from the canonical topological inclusion $X \times_{M} Y \hookrightarrow X \times Y$. The singular space with smooth structure thus obtained is called the fibered product of $X$ and $Y$ over $M$. Since $\pi: X \rightarrow M$ is a singular fiber bundle it follows that $\tau^{*} \pi: X \times_{M} Y \rightarrow Y$ is a singular fiber bundle as well with the same typical fiber $F$. Moreover, this construction satisfies the following universal property. Let $Z$ be a singular space with smooth structure and $f_{1}: Z \rightarrow X$, $f_{2}: Z \rightarrow Y$ be smooth mappings satisfying $\pi \circ f_{1}=\tau \circ f_{2}$. Then there is a unique smooth map $f=\left(f_{1}, f_{2}\right)$ such that the following commutes.

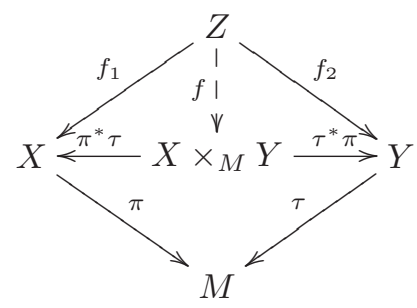

Therefore, in this sense, pull backs exist in the category of singular fiber bundles. There is, in fact, a similar notion of pull backs in the work of Davis [10].

\section{Mechanical connection and Weinstein construction}

Suppose that $Q$ is a Riemannian manifold, and $K$ is a compact Lie group which acts on $Q$ by isometries. Moreover, $Q$ is assumed to be of single isotropy type, i.e., $Q=Q_{(H)}$ where $H$ is an isotropy subgroup of $K$. The $K$-action then induces a Hamiltonian action on the cotangent bundle $T^{*} Q$ by cotangent lifts. This means that the lifted action respects the canonical symplectic form $\Omega=-d \theta$ on $T^{*} Q$ and there is a momentum map $\mu: T^{*} Q \rightarrow \mathfrak{k}^{*}$ given by $\langle\mu(q, p), X\rangle=\theta\left(\zeta_{X}^{T^{*} Q}\right)(q, p)=\left\langle p, \zeta_{X}(q)\right\rangle$ 
where $(q, p) \in T^{*} Q, X \in \mathfrak{k}, \zeta_{X}$ is the fundamental vector field associated to the $K$ action on $Q$, and $\zeta_{X}^{T^{*} Q} \in \mathfrak{X}\left(T^{*} Q\right)$ is the fundamental vector field associated to the cotangent lifted action.

\subsection{Mechanical connection}

Since the $K$-action on $Q$ has only a single isotropy type, the orbit space $Q / K$ is a smooth manifold, and the projection $\pi: Q \rightarrow Q / K$ is a surjective Riemannian submersion with compact fibers. However, the lifted action by $K$ on $T^{*} Q$ is already much more complicated, and the quotient space $\left(T^{*} Q\right) / K$ is only a stratified space in general. Its strata are of the form $\left(T^{*} Q\right)_{(L)} / K$ where $(L)$ is in the isotropy lattice of $T^{*} Q$.

The vertical sub-bundle of $T Q$ with respect to $\pi: Q \rightarrow Q / K$ is $\operatorname{Ver}:=\operatorname{ker} T \pi$. Via the $K$-invariant Riemannian metric we obtain the horizontal sub-bundle as Hor $:=$ $\mathrm{Ver}^{\perp}$. We define the dual horizontal sub-bundle of $T^{*} Q$ as the sub-bundle Hor* consisting of those co-vectors that vanish on all vertical vectors. Likewise, we define the dual vertical sub-bundle of $T^{*} Q$ as the sub-bundle Ver* consisting of those covectors that vanish on all horizontal vectors.

We choose and fix a $K$-invariant inner product on $\mathfrak{k}$. For $X, Y \in \mathfrak{k}$ and $q \in Q$ we define $\mathbb{I}_{q}(X, Y):=\left\langle\zeta_{X}(q), \zeta_{Y}(q)\right\rangle$ and call this the inertia tensor. This gives a non-degenerate pairing on $\mathfrak{k}_{q}^{\perp} \times \mathfrak{k}_{q}^{\perp}$, whence it gives an identification $\check{\mathbb{I}}_{q}: \mathfrak{k}_{q}^{\perp} \rightarrow\left(\mathfrak{k}_{q}^{\perp}\right)^{*}=$ Ann $\mathfrak{k}_{q}$. We use this isomorphism to define a one-form on $Q$ with values in the bundle $\bigsqcup_{q \in Q} \mathfrak{k}_{q}^{\perp}$ by the following:

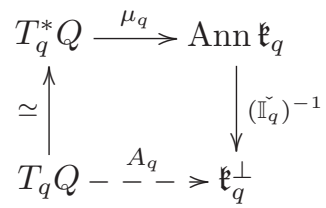

Here the isomorphism $T_{q} Q \stackrel{\simeq}{\rightrightarrows} T_{q}^{*} Q$ is obtained via the $K$-invariant Riemannian metric on $Q$. See Smale [30] or Marsden, Montgomery, and Ratiu [15, section 2]. The form $A$ shall be called the mechanical connection on $Q \rightarrow Q / K$. It has the following properties. It follows from its definition that $T Q \rightarrow \bigsqcup_{q \in Q} \mathfrak{k}_{q}^{\perp},(q, v) \mapsto\left(q, A_{q}(v)\right)$ is equivariant, $\operatorname{ker} A_{q}=T_{q}(K . q)^{\perp}$, and $A_{q}\left(\zeta_{X}(q)\right)=X$ for all $X \in \mathfrak{k}_{q}^{\perp}$.

This means that $A \in \Omega^{1}(Q ; \mathfrak{k})$ given by $A: T Q \rightarrow \mathfrak{k}_{q}^{\perp} \hookrightarrow \mathfrak{k},(q, v) \mapsto A_{q}(v)$ is a principal connection form on the $K$-manifold $Q$ in the sense of Alekseevsky and Michor [2, section 3.1]. According to [2, section 4.6] the curvature form associated to $A$ is defined by

$$
\operatorname{Curv}^{A}:=d A-\frac{1}{2}[A, A]^{\wedge}
$$


where

$$
[\varphi, \psi]^{\wedge}\left(v_{1}, \ldots, v_{l+k}\right):=\frac{1}{k ! l !} \sum_{\sigma} \operatorname{sign} \sigma\left[\varphi\left(v_{\sigma 1}, \ldots, v_{\sigma l}\right), \psi\left(v_{\sigma(l+1)}, \ldots, v_{\sigma(l+k)}\right)\right]
$$

is the graded Lie bracket on $\Omega(Q ; \mathfrak{k}):=\bigoplus_{k=0}^{\infty} \Gamma\left(\Lambda^{k} T^{*} Q \otimes \mathfrak{k}\right)$, and $\varphi \in \Omega^{l}(Q ; \mathfrak{k})$ and $\psi \in \Omega^{k}(Q ; \mathfrak{k})$. The minus in the definition of $\operatorname{Curv}^{A}$ emerges, since we are dealing with left actions. The form $\mathrm{Curv}^{A}$ is called the mechanical curvature.

Proposition 3.1 (Properties of $\mathrm{Curv}^{A}$ ). Let $Z_{1}, Z_{2} \in \mathfrak{k}$ and $v$, w horizontal vector fields on $Q$ with respect to the orbit projection $\pi: Q \rightarrow Q / K$. Then:

(i) $\operatorname{Curv}^{A}\left(\zeta_{Z_{1}}, \zeta_{Z_{2}}\right)(q) \in \mathfrak{k}_{q}$.

(ii) $\operatorname{Curv}^{A}\left(v, \zeta_{Z_{2}}\right)(q)=0$.

(iii) $\operatorname{Curv}^{A}(v, w)(q) \in \mathfrak{k}_{q}^{\perp}$.

Moreover, the form $\operatorname{Curv}^{A}: \Lambda^{2} T Q \rightarrow \mathfrak{k}$ is $K$-equivariant, $\operatorname{Ad}(h) \cdot \operatorname{Curv}^{A}(v, w)(q)=$ $\operatorname{Curv}^{A}(v, w)(q)$ for all $h \in K_{q}$, and $\mathrm{Curv}^{A}$ drops to a well defined form

$$
\operatorname{Curv}_{0}^{A} \in \Omega^{2}\left(Q / K ;\left(\bigsqcup_{q \in Q} \operatorname{Fix}\left(K_{q}\right) \cap \mathfrak{k}_{q}^{\perp}\right) / K\right)
$$

where $\operatorname{Fix}\left(K_{q}\right):=\left\{X \in \mathfrak{k}: K_{q} \subseteq K_{X}\right\}$.

Notice that $\left(\bigsqcup_{q \in Q} \operatorname{Fix}\left(K_{q}\right) \cap \mathfrak{k}_{q}^{\perp}\right) / K$ is a smooth manifold and a topological subspace of the stratified space $\left(\bigsqcup_{q \in Q} \mathfrak{k}_{q}^{\perp}\right) / K$ - see Lemma 4.2.

Proof. Assertion (i) is true since the vertical bundle is integrable: Indeed, $\Phi:=\zeta \circ A$ : $T Q \rightarrow$ Ver defines a (generalized) principal bundle connection on $Q \rightarrow Q / K$ in the sense of Alekseevsky and Michor [2]. As usual, the curvature $R$ associated to $\Phi$ is given by $R(X, Y)=\Phi[X-\Phi X, Y-\Phi Y]$. By [2, Proposition 4.4] $R$ and $\mathrm{Curv}^{A}$ are related by

$$
R=-\zeta \circ \mathrm{Curv}^{A}
$$

whence (i) follows. Using this relation again it follows that $\operatorname{Curv}^{A}\left(v, \zeta_{Z_{2}}\right)(q) \in \mathfrak{k}_{q}$ and this is already sufficient for the purpose of this paper. However, using [2, Proposition 4.7] and the Slice theorem for Riemannian actions the stronger result (ii) is true as well. Assertion (iii) follows also by using the Slice theorem.

Clearly $\mathrm{Curv}^{A}$ is $K$-equivariant. The element $\operatorname{Curv}^{A}(v, w)(q)$ is fixed by all $h \in K_{q}$ since this is true for $q$ and horizontal vectors at $q$ have isotropy at least $K_{q}$. Finally, the last conclusion is an obvious consequence of the above. It is only to notice that $\left(\bigsqcup_{q \in Q} \operatorname{Fix}\left(K_{q}\right) \cap \mathfrak{k}_{q}^{\perp}\right) / K$ really is a smooth fiber bundle over $Q / K$ which, again, is a consequence of the Slice theorem.

We define a pointwise dual $A_{q}^{*}:$ Ann $\mathfrak{k}_{q} \rightarrow \operatorname{Ver}_{q}^{*} \subseteq T_{q}^{*} Q$ by the formula $A_{q}^{*}(\lambda)(v)=$ $\lambda\left(A_{q}(v)\right)$ where $\lambda \in \operatorname{Ann}_{q}$ and $v \in T_{q} Q$. Notice that $A_{q}^{*}\left(\mu_{q}(p)\right)=p$ for all $p \in \operatorname{Ver}_{q}^{*}$ and $\mu_{q}\left(A_{q}^{*}(\lambda)\right)=\lambda$ for all $\lambda \in \operatorname{Ann} \mathfrak{k}_{q}$. 


\subsection{Weinstein bundle construction}

Using the horizontal lift mapping which identifies Hor $\cong\left(Q \times_{Q / K} T(Q / K)\right)$ and the mechanical connection $A$ we obtain an isomorphism

$$
T Q=\text { Hor } \oplus \operatorname{Ver} \longrightarrow\left(Q \times_{Q / K} T(Q / K)\right) \times_{Q} \bigsqcup_{q \in Q} \mathfrak{k}_{q}^{\perp}
$$

of bundles over $Q$. Via the Riemannian structure there is a dual version to this isomorphism, and to save on typing we will abbreviate

$$
\mathcal{W}:=\left(Q \times_{Q / K} T^{*}(Q / K)\right) \times_{Q} \bigsqcup_{q \in Q} \text { Ann } \mathfrak{k}_{q} \cong \text { Hor }^{*} \oplus \text { Ver }^{*} .
$$

To set up some notation for the upcoming proposition, and clarify the picture consider the following stacking of pull-back diagrams. It will be reference point for the whole paper.

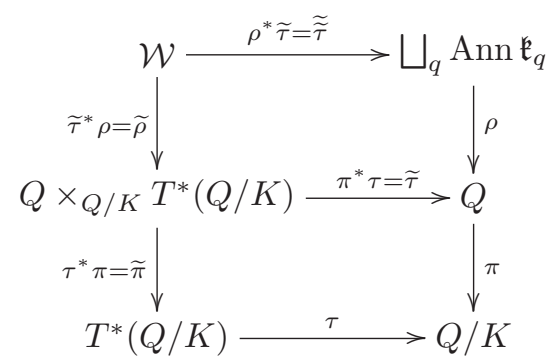

The upper stars in this diagram are, of course, not pull-back stars. It is in fact the transition functions that are being pulled-back, whence the name.

Proposition 3.2 (Symplectic structure on $\mathcal{W}$ ). There is a dual isomorphism

$$
\begin{gathered}
\psi=\psi(A):\left(Q \times{ }_{Q / K} T^{*}(Q / K)\right) \times_{Q} \bigsqcup_{q \in Q} \operatorname{Ann} \mathfrak{k}_{q}=\mathcal{W} \longrightarrow T^{*} Q \\
(q, \eta, \lambda) \longmapsto\left(q, \eta+A(q)^{*} \lambda\right)
\end{gathered}
$$

where we identify elements in $\{q\} \times T_{[q]}^{*}(Q / K)$ with elements in Hor $_{q}^{*}$ via the dual of the inverse of the horizontal lift.

This isomorphism can be used to induce a symplectic form on the connection dependent realization of $T^{*} Q$, namely $\sigma=\psi^{*} \Omega$ where $\Omega=-d \theta$ is the canonical form on $T^{*} Q$. Moreover, there is an explicit formula for $\sigma$ in terms of the chosen connection:

$$
\sigma=(\tilde{\pi} \circ \tilde{\rho})^{*} \Omega^{Q / K}-d \tilde{\tau}^{*} B
$$

where $\Omega^{Q / K}$ is the canonical symplectic form on $T^{*}(Q / K)$, and furthermore $B \in$ $\Omega^{1}\left(\bigsqcup_{q}\right.$ Ann $\left.\mathfrak{k}_{q}\right)$ is given by

$$
B_{(q, \lambda)}\left(v_{1}, \lambda_{1}\right)=\left\langle\lambda, A_{q}\left(v_{1}\right)\right\rangle .
$$


The explicit formula now is

$$
(d B)_{(q, \lambda)}\left(\left(v_{1}, \lambda_{1}\right),\left(v_{2}, \lambda_{2}\right)\right)=\left\langle\lambda, \operatorname{Curv}_{q}^{A}\left(v_{1}, v_{2}\right)\right\rangle+\left\langle\lambda,\left[Z_{1}, Z_{2}\right]\right\rangle-\left\langle\lambda_{2}, Z_{1}\right\rangle+\left\langle\lambda_{1}, Z_{2}\right\rangle
$$

where $(q, \lambda) \in \bigsqcup_{q}$ Ann $\mathfrak{k}_{q},\left(v_{i}, \lambda_{i}\right) \in T_{(q, \lambda)}\left(\bigsqcup_{q}\right.$ Ann $\left.\mathfrak{k}_{q}\right)$ for $i=1,2$, and

$$
v_{i}=\zeta_{Z_{i}}(q) \oplus v_{i}^{\text {hor }} \in \operatorname{Ver}_{q} \oplus \operatorname{Hor}_{q}
$$

is the decomposition into vertical and horizontal part with $Z_{i} \in \mathfrak{k}$.

Furthermore, there clearly is an induced action by $K$ on $\mathcal{W}$. This action is Hamiltonian with momentum mapping

$$
\mu_{A}=\mu \circ \psi: \mathcal{W} \longrightarrow \mathfrak{k}^{*},(q, \eta, \lambda) \longmapsto \lambda,
$$

where $\mu$ is the momentum map $T^{*} Q \rightarrow \mathfrak{k}^{*}$, and $\psi$ is equivariant.

Proof. This proposition is proved in Hochgerner [14, Proposition 5.1]. However, the proof can be considerably simplified using the relation $\sigma=\psi^{*} \Omega=-\psi^{*} d \theta$, and we present this simplification.

Let $\xi \in T \mathcal{W}$ with $T(\tilde{\tau} \circ \tilde{\rho}) \cdot \xi=v \in T Q$ and $w=(q, \eta, \lambda) \in \mathcal{W}$. Then

$$
\left(\psi^{*} \theta\right)_{w}(\xi)=\left\langle\eta+A_{q}^{*}(\lambda), v_{q}\right\rangle=\left((\tilde{\pi} \circ \tilde{\rho})^{*} \theta^{Q / K}\right)_{w}(\xi)+\left(\tilde{\tilde{\tau}}^{*} B\right)_{w}(\xi)
$$

whence $\sigma=(\tilde{\pi} \circ \tilde{\rho})^{*} \Omega^{Q / K}-d \tilde{\tilde{\tau}}^{*} B$. It only remains to compute $d B$ :

$$
\begin{aligned}
&(d B)_{(q, \lambda)}\left(\left(v_{1}, \lambda_{1}\right),\left(v_{2}, \lambda_{2}\right)\right) \\
&=\mathcal{L}_{\left(v_{1}, \lambda_{1}\right)}\left(B\left(v_{2}, \lambda_{2}\right)\right)_{(q, \lambda)}-\mathcal{L}_{\left(v_{2}, \lambda_{2}\right)}\left(B\left(v_{1}, \lambda_{1}\right)\right)_{(q, \lambda)}-B(q, \lambda)\left(\left[\left(v_{1}, \lambda_{1}\right),\left(v_{2}, \lambda_{2}\right)\right]\right) \\
&=\left.\frac{\partial}{\partial t}\right|_{0} B\left(\mathrm{Fl}_{t}^{\left(v_{1}, \lambda_{1}\right)}(q, \lambda)\right)\left(v_{2}, \lambda_{2}\right) \\
& \quad-\left.\frac{\partial}{\partial t}\right|_{0} B\left(\mathrm{Fl}_{t}^{\left(v_{2}, \lambda_{2}\right)}(q, \lambda)\right)\left(v_{1}, \lambda_{1}\right)-\left\langle\lambda, A_{q}\left[v_{1}, v_{2}\right](q)\right\rangle \\
&=\left.\frac{\partial}{\partial t}\right|_{0}\left\langle\mathrm{Fl}_{t}^{\lambda_{1}}(\lambda), A\left(\mathrm{Fl}_{t}^{v_{1}}(q)\right)\left(v_{2}\right)\right\rangle \\
&-\left.\frac{\partial}{\partial t}\right|_{0}\left\langle\mathrm{Fl}_{t}^{\lambda_{2}}(\lambda), A\left(\mathrm{Fl}_{t}^{v_{2}}(q)\right)\left(v_{1}\right)\right\rangle-\left\langle\lambda, A_{q}\left[v_{1}, v_{2}\right](q)\right\rangle \\
&=\left\langle\lambda_{1}(\lambda), Z_{2}\right\rangle-\left\langle\lambda_{2}(\lambda), Z_{1}\right\rangle+\left\langle\lambda, \mathcal{L}_{v_{1}}\left(A\left(v_{2}\right)\right)_{q}-\mathcal{L}_{v_{2}}\left(A\left(v_{1}\right)\right)_{q}-A_{q}\left[v_{1}, v_{2}\right](q)\right\rangle \\
&=\left\langle\lambda_{1}(\lambda), Z_{2}\right\rangle-\left\langle\lambda_{2}(\lambda), Z_{1}\right\rangle+\left\langle\lambda, d A_{q}\left(v_{1}, v_{2}\right)\right\rangle \\
&=\left\langle\lambda_{1}(\lambda), Z_{2}\right\rangle-\left\langle\lambda_{2}(\lambda), Z_{1}\right\rangle+\left\langle\lambda, \operatorname{Curv}_{q}^{A}\left(v_{1}, v_{2}\right)\right\rangle+\left\langle\lambda,\left[Z_{1}, Z_{2}\right]\right\rangle .
\end{aligned}
$$

\section{Gauged Poisson reduction}

Let $H$ be a closed subgroup of our compact Lie group $K$. Let $S$ be a smooth manifold, and $a: H \times S \rightarrow S,(h, s) \mapsto h \cdot s$ a left action. We will be mostly interested in the case 
where $S$ is a slice for the $K$-action on $Q$, and $a$ is the (trivial) slice representation. Consider the left actions $l, r, t: H \times S \times K \rightarrow S \times K$ which are defined by

$$
\begin{gathered}
l:(h, s, k) \longmapsto(s, h k), \quad r:(h, s, k) \longmapsto\left(s, k h^{-1}\right), \\
\text { and } t:(h, s, k) \longmapsto\left(h \cdot s, k h^{-1}\right) .
\end{gathered}
$$

The following lemma is elementary on the one hand as its computations are straightforward. However, it is also tricky on the other hand since it involves a choice of sign in the definition of the fundamental vector field from section 1, and there are many possibilities to get confused. Note also that we use the left multiplication to trivialize $T^{*} K=K \times \mathfrak{k}^{*}$.

Lemma 4.1 (Cotangent lifted actions). The lifted actions of $H$ on $T^{*}(S \times K)=$ $T^{*} S \times K \times \mathfrak{k}^{*}$ corresponding to $l, r, t$ are given by

$$
\begin{aligned}
T^{*} S \times K \times \mathfrak{k}^{*} & \longrightarrow T^{*} S \times K \times \mathfrak{k}^{*} \\
T^{*} l_{h}:(s, p, k, \eta) & \longmapsto(s, p, h k, \eta) \\
T^{*} r_{h}:(s, p, k, \eta) & \longmapsto\left(s, p, k h^{-1}, \mathrm{Ad}^{*}(h) \cdot \eta\right) \\
T^{*} t_{h}:(s, p, k, \eta) & \longmapsto\left(h \cdot s, T_{s}^{*} a_{h} \cdot p, k h^{-1}, \operatorname{Ad}^{*}(h) \cdot \eta\right)
\end{aligned}
$$

respectively, and where $\operatorname{Ad}^{*}(h) \cdot \eta:=\eta \circ \operatorname{Ad}\left(h^{-1}\right)$. Moreover, these lifted actions are Hamiltonian with respect to the canonical exact symplectic form on $T^{*}(S \times K)=$ $T^{*} S \times K \times \mathfrak{k}^{*}$, and the corresponding momentum maps are given by

$$
\begin{aligned}
T^{*} S \times K \times \mathfrak{k}^{*} & \longmapsto \mathfrak{h}^{*} \\
J_{l}:(s, p, k, \eta) & \longmapsto\left(\operatorname{Ad}^{*}(k) \cdot \eta\right) \mid \mathfrak{h} \\
J_{r}:(s, p, k, \eta) & \longmapsto-\eta \mid \mathfrak{h} \\
J_{t}:(s, p, k, \eta) & \longmapsto \mu(s, p)-\eta \mid \mathfrak{h}
\end{aligned}
$$

where $\mu: T^{*} S \rightarrow \mathfrak{h}^{*}$ is the canonical equivariant momentum map with respect to the Hamiltonian action $T^{*} a: H \times T^{*} S \rightarrow T^{*} S$.

Proof. The point here is the choice of sign in the definition of the fundamental vector field mapping $\zeta$ in section 1 . Note also that the cotangent bundle momentum map $\mu: T^{*} S \rightarrow \mathfrak{h}^{*}$ is given by $\langle\mu(s, p), X\rangle=\theta\left(\zeta_{X}^{a}\right)(s, p)$ where $\theta$ is the Liouville form on $T^{*} S$, and $\Omega=-d \theta$ is the cotangent bundle symplectic form.

Let us introduce the abbreviation $E:=\bigsqcup_{q \in Q}$ Ann $\mathfrak{k}_{q}$.

Lemma 4.2. The natural projection $\rho: E \rightarrow Q$ is a smooth fiber bundle with typical fiber Ann $\mathfrak{h}$, were $H$ is an isotropy subgroup of $K$ and $\mathfrak{h}$ is its Lie algebra. 
Proof. We trivialize at an arbitrary point $q_{0} \in Q$. We may assume $K_{q_{0}}=H$. Let $U \subseteq Q$ be a tube around $K . q_{0}$ such that $U \cong S \times K / H$ as $K$-spaces, where $S$ is a slice at $q_{0}$. Then it is possible to trivialize as

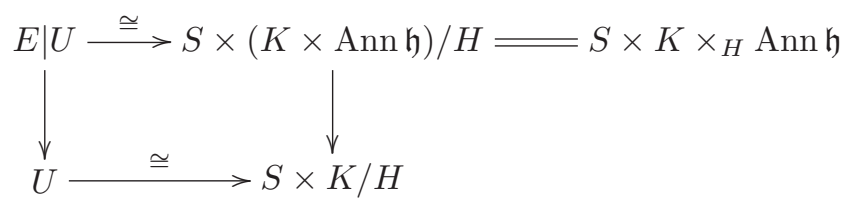

with trivializing map given by

$$
S \times K \times_{H} \text { Ann } \mathfrak{h} \longrightarrow E \mid U, \quad(s,[(k, \lambda)]) \longmapsto\left(k \cdot s, \operatorname{Ad}^{*}(k)(\lambda)\right)
$$

where $\operatorname{Ad}^{*}(k)(\lambda):=\operatorname{Ad}\left(k^{-1}\right)^{*}(\lambda)$. This map is well-defined and smooth with inverse given by

$$
(q, \lambda)=\left(k \cdot s, \operatorname{Ad}^{*}(k)\left(\lambda_{0}\right)\right) \longmapsto\left(s,\left[\left(k, \lambda_{0}\right)\right]\right)
$$

which is well-defined and smooth as well. Notice furthermore that the trivializing map is $K$-equivariant with respect to the $K$-action on $S \times K \times_{H}$ Ann $\mathfrak{h}$ given by $g \cdot(s,[(k, \lambda)])=(s,[(g k, \lambda)])$. Indeed, this follows immediately from Lemma 4.1.

Lemma 4.3. Let $E:=\bigsqcup_{q \in Q} \operatorname{Ann}_{q}$.

(i) Let $U \subseteq Q$ be a trivializing neighborhood for $\rho: E \rightarrow Q$ as in the proof of Lemma 4.2. Then $E \mid U$ is $K$-invariant, and if $(L)$ is an element of the isotropy lattice for the $K$-action on $E$ then the corresponding stratum is trivialized as

$$
(E \mid U)_{(L)} \cong S \times K \times_{H}(\operatorname{Ann} \mathfrak{h})_{\left(L_{0}\right)^{H}}
$$

where $L_{0} \subseteq H$ is an isotropy subgroup for the $H$-action conjugate to $L$ in $K$, and $\left(L_{0}\right)^{H}$ is the conjugacy class of $L_{0}$ in $H$. Moreover, the strata of Ann $\mathfrak{h} / H$ are of the form $(\text { Ann } \mathfrak{h})_{\left(L_{0}\right)^{H}} / H$.

(ii) The induced mapping $\rho_{0}: E / K \rightarrow Q / K$ is a singular fiber bundle with typical fiber Ann $\mathfrak{h} / H$ in the sense of Definition 2.13.

Proof. Let $q_{0} \in Q$ with $K_{q_{0}}=H$. Then

$$
\left(q_{0}, \lambda\right) \in\left(\bigsqcup_{q \in Q} \operatorname{Ann} \mathfrak{k}_{q}\right)_{(L)}=E_{(L)}
$$

if and only if

$$
\lambda \in \text { Ann } \mathfrak{h} \quad \text { and } \quad H \cap K_{\lambda}=H_{\lambda}=L_{0} \sim L \text { within } K
$$

which is true if and only if

$$
\lambda \in(\operatorname{Ann} \mathfrak{h})_{\left(L_{0}\right)^{H}}
$$


where $L_{0}$ is a subgroup of $H$ conjugate to $L$ within $K$. Notice that it follows from the Slice Theorem for Riemannian actions that $(\operatorname{Ann} \mathfrak{h})_{\left(L_{0}\right)^{H}}$ is a smooth manifold, e.g., Palais and Terng [22]. Therefore, also the second assertion follows.

Theorem 4.4. There is a stratified isomorphism of stratified bundles over $Q / K$, in the sense of Definition 2.13,

$$
\begin{gathered}
\psi_{0}^{-1}=\psi_{0}^{-1}(A):\left(T^{*} Q\right) / K \longrightarrow T^{*}(Q / K) \times_{Q / K}\left(\bigsqcup_{q \in Q} \operatorname{Ann}_{q}\right) / K=: W, \\
{[(q, p)] \longmapsto\left(C^{*}(q, p),[(q, \mu(q, p))]\right)}
\end{gathered}
$$

where the stratification was suppressed. Here

$$
C^{*}: T^{*} Q \rightarrow \text { Hor* }^{*} T^{*}(Q / K)
$$

is constructed as the pointwise dual to the horizontal lift mapping $C: T(Q / K) \times{ }_{Q / K} Q$ $\rightarrow$ Hor $\subseteq T Q,([q], v ; q) \rightarrow C_{q}(v)$ associated to the connection $A \in \Omega^{1}(Q ; \mathfrak{k})$.

If $(L)$ is an isotropy class of the $K$-action on $T^{*} Q$, then $\psi_{0}^{-1}$ maps the isotropy stratum $\left(T^{*} Q\right)_{(L)} / K$ onto

$$
T^{*}(Q / K) \times_{Q / K}\left(\bigsqcup_{q \in Q} \operatorname{Ann} \mathfrak{k}_{q}\right)_{(L)} / K=: W_{(L)} .
$$

Moreover, the natural projection

$$
\tilde{\rho}_{0}^{(L)}: W_{(L)} \rightarrow T^{*}(Q / K)
$$

is a smooth fiber bundle with typical fiber of the form $(\text { Ann } \mathfrak{h})_{\left(L_{0}\right)^{H}} / H$. Here $L_{0} \subseteq H$ is an isotropy subgroup for the $H$-action conjugate to $L$ in $K$, and $\left(L_{0}\right)^{H}$ is the conjugacy class of $L_{0}$ in $H$.

Therefore, $\tilde{\rho}_{0}: W \rightarrow T^{*}(Q / K)$ is a singular fiber bundle in the sense of Definition 2.13.

In the case that $K$ acts on $Q$ freely the first assertion of the above theorem can also be found in Cendra, Holm, Marsden, Ratiu [8]. Following Ortega and Ratiu [20, section 6.6.12] the above constructed interpretation $W$ of $\left(T^{*} Q\right) / K$ is called Weinstein space referring to Weinstein [32] where this universal construction first appeared.

Proof. We consider first the map

$$
\begin{gathered}
\varphi_{0}=\varphi_{0}(A):(T Q) / K \longrightarrow T(Q / K) \times_{Q / K}\left(\bigsqcup_{q \in Q} \mathfrak{k}_{q}^{\perp}\right) / K, \\
{[(q, v)] \longmapsto\left(T \pi(q, v),\left[\left(q, A_{q} v\right)\right]\right)}
\end{gathered}
$$


the pointwise dual of whose inverse will be $\psi_{0}^{-1}$.

The spaces $T Q$ and $(T Q) / K$ are stratified into isotropy types. Since the base $Q$ is stratified as consisting only of a single stratum, the equivariant foot point projection map $\tau: T Q \rightarrow Q$ is trivially a stratified map. Using the Slice Theorem on the base $Q$ it is easy to see that both $(T Q) / K \rightarrow Q / K$ and the projection $\left(\bigsqcup_{q \in Q} \mathfrak{k}_{q}^{\perp}\right) / K \rightarrow Q / K$ are singular bundle maps in the sense of Definition 2.13. Hereby $\left(\bigsqcup_{q \in Q} \mathfrak{k}_{q}^{\perp}\right) / K$ is stratified into isotropy types. According to Davis [10] or also Subsection 2.5 pullbacks are well defined in the category of stratified spaces and thus it makes sense to define $T(Q / K) \times_{Q / K}\left(\bigsqcup_{q \in Q} \mathfrak{k}_{q}^{\perp}\right) / K$ as a stratified space with smooth structure.

The map $\varphi_{0}$ is well defined: indeed, for $(q, v) \in T Q$ and $k \in K$ we have

$$
T \pi(k \cdot q, k \cdot v)=\left(\pi(k \cdot q), T_{k \cdot q} \pi\left(T_{q} l_{k}(v)\right)\right)=\left(\pi(q), T_{q}\left(\pi \circ l_{k}\right)(v)\right)=T_{q} \pi(v),
$$

and $[(k \cdot q, A(k \cdot q, k \cdot v))]=[(q, A(q, v))]$ by equivariance of $A$. It is clearly continuous as a composition of continuous maps. Moreover, since $C^{\infty}((T Q) / K)=C^{\infty}(T Q)^{K}$ by Example 2.10 it follows that $\varphi_{0}$ is a smooth map of singular spaces.

We claim that $\varphi_{0}$ maps strata onto strata, and moreover we have the formula

$$
\varphi_{0}\left((T Q)_{(L)} / K\right)=T(Q / K) \times_{Q / K}\left(\bigsqcup_{q \in Q} \mathfrak{k}_{q}^{\perp}\right)_{(L)} / K .
$$

Indeed, consider $(q, v) \in(T Q)_{(L)}$, that is $H \cap K_{v}=L^{\prime} \sim L$ where $H=K_{q}$. The notation $L^{\prime} \sim L$ means that $L^{\prime}$ is conjugate to $L$ within $K$. Now we can decompose $v$ as $v=v_{0} \oplus \zeta_{X}(q) \in \operatorname{Hor}_{q} \oplus T_{q}(K \cdot q)$ for some appropriate $X \in \mathfrak{k}$. Since $Q$ consists only of a single isotropy type we have $T_{q} Q=T_{q} Q_{H}+T_{q}(K \cdot q)$ - which is not a direct sum decomposition. As usual, $Q_{H}=\left\{q \in Q: K_{q}=H\right\}$. This shows that $v_{0} \in T_{q} Q_{H}$, since $\operatorname{Hor}_{q}=T_{q}(K \cdot q)^{\perp} \subseteq T_{q} Q_{H}$, and hence $H \subseteq K_{v_{0}}$. By equivariance of $A$ it follows that

$$
K_{q} \cap K_{v}=H \cap K_{v_{0}} \cap K_{\zeta_{X}(q)}=H \cap K_{\zeta_{X}(q)}=H \cap K_{A(q, v)}
$$

which is independent of the horizontal component. Hence the claim. The restriction of $\varphi_{0}$ to any stratum clearly is smooth as a composition of smooth maps.

Since $A_{q}\left(\zeta_{X}(q)\right)=X$ for $X \in \mathfrak{k}_{q}^{\perp}$ we can write down an inverse as

$$
\varphi_{0}^{-1}:([q], v ;[(q, X)]) \rightarrow\left[\left(q, C_{q}(v)+\zeta_{X}(q)\right)\right]
$$

and again it is an easy matter to notice that this map is well defined, continuous, and smooth on each stratum. Again, it follows from the definition of the smooth structures on the respective spaces that $\varphi_{0}^{-1}$ is smooth.

It makes sense to define the dual $\psi_{0}^{-1}$ of the inverse map $\varphi_{0}^{-1}$ in a point wise 
manner, and it only remains to compute this map.

$$
\begin{aligned}
\left\langle\psi_{0}^{-1}[(q, p)],([q], v ;[(q, X)])\right\rangle & =\left\langle[(q, p)],\left[\left(q, C_{q}(v)+\zeta_{X}(q)\right)\right]\right\rangle \\
& =\left\langle p, C_{q}(v)\right\rangle+\left\langle p, \zeta_{X}(q)\right\rangle \\
& =\left\langle C^{*}(q, p), v\right\rangle+\langle\mu(q, p), X\rangle \\
& =\left\langle\left(C^{*}(q, p),[(q, \mu(q, p))]\right),([q], v ;[(q, X)])\right\rangle
\end{aligned}
$$

where we used the $K$-invariance of the dual pairing over $Q$.

Finally, $\psi_{0}^{-1}$ is an isomorphism of singular Poisson spaces: note first that the identifying map $\mathcal{W} / K \rightarrow W,[(q ;[q], \eta ; q, \lambda)] \mapsto([q], \eta ;[(q, \lambda)])$ is well-defined because $K_{q}$ acts trivially on $\operatorname{Hor}_{q}^{*}=T_{[q]}^{*}(Q / K) \ni \eta$ which in turn is due to the fact that all points of $Q$ are regular. Moreover, by the universal property for singular pull back bundles from subsection 2.5 it is obvious that this map $\mathcal{W} / K \rightarrow W$ is smooth and has a smooth inverse. The quotient Poisson bracket is well-defined since $C^{\infty}(\mathcal{W})^{K} \subseteq$ $C^{\infty}(\mathcal{W})$ is a Poisson sub-algebra. The statement now follows because the diagram

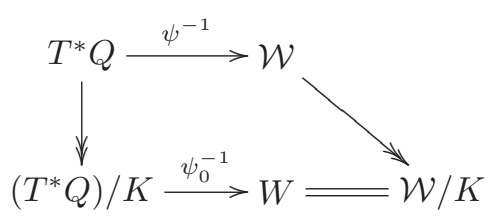

is commutative, and composition of top and down-right arrow is Poisson and the left vertical arrow is surjective.

Using Lemma 4.3 together with Subsection 2.3 the global description of $\mathcal{W} / K \cong W$ as a fibered product thus follows.

Next we shall construct a connection on $\rho: E \rightarrow Q$ which will provide a connection on $\tilde{\rho}: \mathcal{W} \rightarrow Q \times_{Q / K} T^{*}(Q / K)$. Recall the mechanical connection $A \in \Omega^{1}(Q ; \mathfrak{k})$ from section 3. Consider the embedding

$$
\iota: E \longrightarrow T^{*} Q, \quad(q, \lambda) \longmapsto\left(q, A_{q}^{*}(\lambda)\right) .
$$

On $\varrho: T^{*} Q \rightarrow Q$ we choose the canonical (with respect to the metric) linear connection $\Phi(\varrho): T T^{*} Q \rightarrow V(\varrho)$. Consider the following diagram

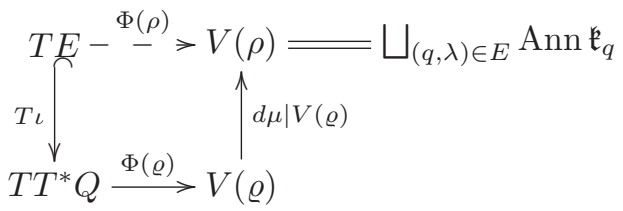

which induces a linear connection $\Phi(\rho)$ on $\rho: E \rightarrow Q$. 
Lemma 4.5. The diagram (2) is well-defined.

Proof. We have to show that $d \mu \mid V(\varrho)$ takes values in $V(\rho)=\bigsqcup_{(q, \lambda) \in E}$ Ann $\mathfrak{k}_{q}$. Since

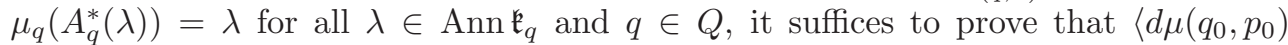
$\left.\left(\xi_{\left(q_{0}, p_{0}\right)}\right), X\right\rangle=0$ for fixed $\left(q_{0}, p_{0}\right) \in T^{*} Q, \xi \in \Gamma(V(\varrho))$, and $X \in \mathfrak{k}_{q_{0}}$. Now, for arbitrary $(q, p) \in T^{*} Q$,

$$
\begin{aligned}
\left\langle d \mu(q, p)\left(\xi_{(q, p)}\right), X\right\rangle & =\Omega_{(q, p)}\left(\zeta_{X}^{T^{*} Q}, \xi\right) \\
& =-\left(\zeta_{X}^{T^{*} Q} \cdot \theta(\xi)\right)(q, p)+\left(\xi \cdot \theta\left(\zeta_{X}^{T^{*} Q}\right)\right)(q, p)+\theta\left(\left[\zeta_{X}^{T^{*} Q}, \xi\right]\right)(q, p) .
\end{aligned}
$$

We have

$$
\theta(\xi)(q, p)=\left\langle p, T_{(q, p)} \varrho \cdot \xi_{(q, p)}\right\rangle=0
$$

for all $(q, p) \in T^{*} Q$ and $\xi \in \Gamma(V(\varrho))$, so $\theta(\xi)=0$. Next we find

$$
\theta\left(\zeta_{X}^{T^{*} Q}\right)\left(q_{0}, p\right)=\left\langle p, T_{\left(q_{0}, p\right)} \varrho \cdot \zeta_{X}^{T^{*} Q}\left(q_{0}, p\right)\right\rangle=\left\langle p, \zeta_{X}\left(q_{0}\right)\right\rangle=0
$$

for all $p \in T_{q_{0}}^{*} Q$ since $X \in \mathfrak{k}_{q_{0}}$ by assumption. Since $\xi$ takes values in the vertical subbundle $V(\varrho)$ and vertical bundles are integrable its flow preserves fibers of $\varrho$, that is $\mathrm{Fl}_{t}^{\xi}\left(q_{0}, p_{0}\right)=\left(q_{0}, p_{t}\right)$ for some curve $p_{t}$ in $T_{q_{0}}^{*} Q$. Therefore,

$$
\left(\xi \cdot \theta\left(\zeta_{X}^{T^{*} Q}\right)\right)\left(q_{0}, p_{0}\right)=\left.\frac{\partial}{\partial t}\right|_{0}\left\langle p_{t}, \zeta_{X}\left(q_{0}\right)\right\rangle=\left.\frac{\partial}{\partial t}\right|_{0} 0=0 .
$$

Finally,

$$
\theta\left(\left[\zeta_{X}^{T^{*} Q}, \xi\right]\right)(q, p)=\left\langle p, T_{(q, p)} \varrho \cdot\left[\zeta_{X}^{T^{*} Q}, \xi\right](q, p)\right\rangle=0
$$

for all $(q, p) \in T^{*} Q$, since the fact that $\zeta_{X}^{T^{*} Q}$ and $\zeta_{X}$ are $\varrho$-related as well as $\xi$ and 0 implies that $\left[\zeta_{X}^{T^{*} Q}, \xi\right]$ and $\left[\zeta_{X}, 0\right]=0$ are $\varrho$-related, i.e., $T_{(q, p)} \varrho .\left[\zeta_{X}^{T^{*} Q}, \xi\right](q, p)=0$. This completes the proof.

Via the pullback construction (see Diagram (1)) this also induces a linear connection on $\tilde{\rho}: \mathcal{W} \rightarrow Q \times_{Q / K} T^{*}(Q / K)$. We denote this connection by $\tilde{A}: T \mathcal{W} \rightarrow V(\tilde{\rho})$. Notice that $V_{(q, \eta, \lambda)}(\tilde{\rho})=$ Ann $\mathfrak{k}_{q}$ by construction.

The connection $\widetilde{A}$ and the momentum map $\mu_{A}:=\mu \circ \psi: \mathcal{W} \rightarrow \mathfrak{k}^{*}$ are related by

$$
\widetilde{A}(q, \eta, \lambda)(\xi)=d \mu_{A}(q, \eta, \lambda)(\xi),
$$

where $\xi \in T_{(q, \eta, \lambda)} \mathcal{W}$, and $(q, \eta, \lambda)$ is short-hand for $(q ;[q], \eta ; q, \lambda) \in \mathcal{W}$.

We will use the connection $\widetilde{A}$ to decompose an arbitrary vector $\xi \in T_{(q, \eta, \lambda)} \mathcal{W}$ as

$$
\xi=\left(v(q) ; \eta^{\prime}([q], \eta) ; v_{1}(q), \nu(q, \lambda)\right),
$$

where $\nu(q, \lambda)=\widetilde{A}(q, \eta, \lambda)(\xi)$ is independent of $\eta$. Notice also that $v_{1}(q)=v(q)$ by the pullback property. Further we can decompose $v(q) \in T_{q} Q$ according to

$$
v(q)=v(q)^{\operatorname{hor}(\pi)}+\zeta_{Z}(q) \in H_{q}(\pi) \oplus V_{q}(\pi)
$$


with respect to the connection $A$ on $\pi: Q \rightarrow Q / K$. The same can be done with $\eta^{\prime}([q], \eta) \in T_{([q], \eta)}\left(T^{*}(Q / K)\right)$ as

$$
\eta^{\prime}([q], \eta)=\eta^{\prime}([q], \eta)^{\operatorname{hor}(\tau)}+\eta^{\prime}([q], \eta)^{\operatorname{ver}(\tau)} \in H_{([q], \eta)}(\tau) \oplus V_{([q], \eta)}(\tau)
$$

with respect to the canonical connection on $\tau: T^{*}(Q / K) \rightarrow Q / K$ which comes from the induced metric on $Q / K$. Notice that we have $\eta^{\prime}([q], \eta)^{\operatorname{hor}(\tau)}=v(q)^{\operatorname{hor}(\pi)}$ by the pullback property.

Definition 4.6 (Vertical differentiation on $\tilde{\rho}: \mathcal{W} \rightarrow Q \times_{Q / K} T^{*}(Q / K)$ ). Let $\nu \in$ $V_{(q, \eta, \lambda)}(\tilde{\rho})=\operatorname{Ann} \mathfrak{k}_{q}$ and $F \in C^{\infty}(\mathcal{W})$. We define

$$
d_{v} F(q, \eta, \lambda)(\nu):=\left.\frac{\partial}{\partial t}\right|_{0} F(q, \eta, \lambda+t \nu)
$$

to be the vertical derivative of $F$ at $(q, \eta, \lambda)$.

Definition 4.7 (Covariant differentiation on $\tilde{\rho}: \mathcal{W} \rightarrow Q \times_{Q / K} T^{*}(Q / K)$ ). Let $\chi:=1-\widetilde{A}: T \mathcal{W} \rightarrow H(\tilde{\rho})$ denote the horizontal projection with respect to $\tilde{\rho}$. The covariant derivative of $F \in C^{\infty}(\mathcal{W})$ is defined as $d_{\widetilde{A}} F:=d F \circ \chi$.

Lemma 4.8. Let $F \in C^{\infty}(\mathcal{W})^{K}$, and decompose the Hamiltonian vector field of $F$ at $(q, \eta, \lambda) \in \mathcal{W}$ as

$$
\nabla_{F}^{\sigma}(q, \eta, \lambda)=\left(v(q) ; \eta^{\prime}([q], \eta) ; v(q), \nu(q, \lambda)\right)
$$

with $\nu(q, \lambda)=\widetilde{A}(q, \eta, \lambda)\left(\nabla_{F}^{\sigma}(q, \eta, \lambda)\right)$ according to above. Here $\sigma$ is the symplectic structure on $\mathcal{W}$ from Proposition 3.2. Then, $\nu(q, \lambda)=0$.

Proof. This is a consequence of Noether's Theorem. We have

$$
\nu(q, \lambda)=\widetilde{A}(q, \eta, \lambda)\left(\nabla_{F}^{\sigma}(q, \eta, \lambda)\right)=d \mu_{A}(q, \eta, \lambda)\left(\nabla_{F}^{\sigma}(q, \eta, \lambda)\right)=0,
$$

since $\mu_{A}$ is constant along flow lines of Hamiltonian vector fields of invariant functions.

Lemma 4.9. Let $F \in C^{\infty}(\mathcal{W})^{K}$, and decompose the Hamiltonian vector field of $F$ at $(q, \eta, \lambda) \in \mathcal{W}$ as

$$
\nabla_{F}^{\sigma}(q, \eta, \lambda)=\left(v(q) ; \eta^{\prime}([q], \eta) ; v(q), 0\right) .
$$

Then,

$$
\eta^{\prime}([q], \eta)=\left(\left(\Omega_{[[q], \eta)}^{Q / K}\right)^{\vee}\right)^{-1}\left(d_{\widetilde{A}} F(q, \eta, \lambda)\right),
$$

where we consider $d_{\widetilde{A}} F(q, \eta, \lambda)$ as an element of $T_{([q], \eta)}^{*}\left(T^{*}(Q / K)\right)$ via the dual of the horizontal lift mapping. 
Recall that $\Omega^{Q / K}$ denotes the canonical symplectic form on $T^{*}(Q / K)$.

Proof. For the purpose of the lemma we may assume that the vertical part of the first entry vanishes, i.e, $A \cdot T(\widetilde{\tau} \circ \widetilde{\rho}) \cdot \nabla_{F}^{\sigma}=0$ with notation as in diagram (1). Thus we may simplify the problem by assuming that $F \in C^{\infty}(\mathcal{W})^{K}$ factors through $\tilde{\pi} \circ \tilde{\rho}$, i.e., there is a smooth map $f: T^{*}(Q / K) \rightarrow \mathbb{R}$ such that $F=f \circ \tilde{\pi} \circ \tilde{\rho}: \mathcal{W} \rightarrow \mathbb{R}$. Then, $d_{\widetilde{A}} F(q, \eta, \lambda)=d_{\widetilde{A}} F(q, \eta, 0)=d f((\tilde{\pi} \circ \tilde{\rho})(q, \eta, 0))=d f([q], \eta)$. Now, the projection

$$
Q \times_{Q / K} T^{*}(Q / K)=\mu_{A}^{-1}(0) \longrightarrow \mu_{A}^{-1}(0) / K=T^{*}(Q / K)
$$

is a Poisson morphism. Therefore, we find

$$
\begin{aligned}
\eta^{\prime}([q], \eta) & =T_{(q, \eta, 0)}(\tilde{\pi} \circ \tilde{\rho}) \cdot \nabla_{F}^{\sigma}(q, \eta, 0)=\nabla_{f}^{Q / K}((\tilde{\pi} \circ \tilde{\rho})(q, \eta, 0)) \\
& =\left(\left(\Omega_{([q], \eta)}^{Q / K}\right)^{\vee}\right)^{-1}(d f([q], \eta))=\left(\left(\Omega_{([q], \eta)}^{Q / K}\right)^{\vee}\right)^{-1}\left(d_{\widetilde{A}} F(q, \eta, 0)\right)
\end{aligned}
$$

which gives the assertion.

Lemma 4.10. Let $F \in C^{\infty}(\mathcal{W})^{K}$, and decompose the Hamiltonian vector field of $F$ at $(q, \eta, \lambda) \in \mathcal{W}$ as

$$
\nabla_{F}^{\sigma}(q, \eta, \lambda)=\left(v(q) ; \eta^{\prime}([q], \eta) ; v(q), 0\right) .
$$

Via the connection $A \in \Omega^{1}(Q ; \mathfrak{k})$ we can write

$$
v(q)=v(q)^{\operatorname{hor}(\pi)}+\zeta_{Z}(q) \in H_{q}(\pi) \oplus V_{q}(\pi) .
$$

Then $Z=-d_{v} F(q, \eta, \lambda) \in \mathfrak{k}_{q}^{\perp}$, and, moreover, $\operatorname{Ad}(h) \cdot Z=Z$ for all $h \in K_{q}$.

Proof. We work in tube coordinates around $q \in Q$. Thus let $S$ be a slice through $q$ for the $K$-action on $Q$ such that $U \cong S \times K / H$ as $K$-spaces where $U$ is a tube around $K \cdot q$ and $H=K_{q}$. Then we have a $K$-equivariant isomorphism of symplectic manifolds

$$
\mathcal{W} \mid U \cong T^{*} S \times K \times_{H} \text { Ann } \mathfrak{h} \cong T^{*} S \times T^{*} K / / T_{0} T^{*} R(H)
$$

where the right hand side carries the obvious symplectic structure. Here $T^{*} R(H)$ is the cotangent lifted action of the right multiplication of $H$ on $K$, and we use left multiplication to trivialize $T^{*} K=K \times \mathfrak{k}^{*}$. This follows from Lemma 4.1 and an argument similar as in the proof of Lemma 4.2. In particular, note that (as in Hochgerner [14, section 4]) the isomorphism is symplectic since it comes as the cotangent lift of a diffeomorphism of the base spaces. Since we already know that the part of the Hamiltonian vector field of $F$ which is tangent to $T^{*} S$ is given by the local coordinates of $\left(v(q)^{\operatorname{hor}(\pi)}, \eta^{\prime}([q], \eta)\right)$, we may further reduce the problem to considering a function $F \in C^{\infty}\left(K \times_{H} A n n \mathfrak{h}\right)^{K}=C^{\infty}(\operatorname{Ann} \mathfrak{h} / H)$. This identification is due to Lemma 4.3(ii). Now, referring again to Lemma 4.1 we have

$$
K \times_{H} \operatorname{Ann} \mathfrak{h}=T^{*} K / /{ }_{0} T^{*} R(H),
$$


where $T^{*} R(H)$ is the cotangent lifted action of the right multiplication of $H$ on $K$, and where we use left multiplication to trivialize $T^{*} K=K \times \mathfrak{k}^{*}$. Thus, there exists a function $F_{1} \in C^{\infty}\left(T^{*} K\right)=C^{\infty}\left(K \times \mathfrak{k}^{*}\right)$ that is $T^{*} L(K)$-invariant ( $L$ denotes the left multiplication on $K)$ and $T^{*} R(H)$-invariant such that the following diagram commutes:

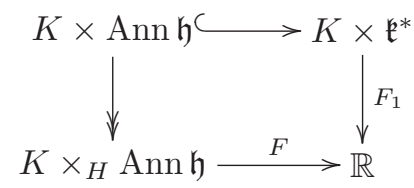

We choose local cotangent bundle coordinates $a_{i}, b_{i}$ where $i=1, \ldots, m$ on $T^{*} K=$ $K \times \mathfrak{k}^{*}$ such that $b_{1}, \ldots, b_{l}$ are coordinates on $\mathfrak{h}^{*}, b_{l+1}, \ldots, b_{m}$ are coordinates on Ann $\mathfrak{h}$, and such that $\frac{\partial}{\partial a_{1}}, \ldots, \frac{\partial}{\partial a_{l}}$ are a basis of $\mathfrak{h}$, and $\frac{\partial}{\partial a_{l+1}}, \ldots, \frac{\partial}{\partial a_{m}}$ are a basis of $\mathfrak{h}^{\perp}$. Then for the canonical Poisson bracket on $T^{*} K$ we obtain

$$
\begin{aligned}
-\nabla_{F_{1}}^{T^{*} K}(e, \lambda) & =\left\{F_{1}, \cdot\right\}^{T^{*} K}(e, \lambda)= \\
& =\sum_{i=1}^{m}\left(\frac{\partial F_{1}}{\partial b_{i}}(e, \lambda) \frac{\partial}{\partial a_{i}}-\frac{\partial F_{1}}{\partial a_{i}}(e, \lambda) \frac{\partial}{\partial b_{i}}\right)=\sum_{i=l+1}^{m} \frac{\partial F_{1}}{\partial b_{i}}(e, \lambda) \frac{\partial}{\partial a_{i}} \in \mathfrak{h}^{\perp}
\end{aligned}
$$

which is the vertical derivative of $F_{1}$ identified with an element of $\mathfrak{h}^{\perp}$ through choice of a basis. Since the projection $K \times \operatorname{Ann} \mathfrak{h} \rightarrow K \times_{H}$ Ann $\mathfrak{h}$ is Poisson, Hamiltonian vector fields project to Hamiltonian vector fields, i.e., $-\nabla_{F_{1}}^{T^{*} K}(e, \lambda)$ projects to

$$
-\nabla_{F}^{K \times{ }_{H} \operatorname{Ann} \mathfrak{h}}[(e, \lambda)]=-Z .
$$

Therefore, the Hamiltonian vector field of $F$ on $K \times_{H}$ Ann $\mathfrak{h}$ is $\left(d_{v} F, 0\right)$ which is tangent to the $K / H$-factor. Thus $-Z=d_{v} F(q, \eta, \lambda) \in \mathfrak{k}_{q}^{\perp}$.

To see the second assertion let $h \in H$ and notice that

$$
\operatorname{Ad}(h) \cdot \nabla_{F_{1}}^{T^{*} K}(e, \lambda)=T_{h} R_{h^{-1}} \cdot T_{e} L_{h} \cdot \nabla_{F_{1}}^{T^{*} K}(e, \lambda)=\nabla_{F_{1}}^{T^{*} K}\left(e, \operatorname{Ad}^{*}(h) \cdot \lambda\right)
$$

since $F_{1}$ is both $T^{*} L(H)$ - and $T^{*} R(H)$-invariant. Let $\pi_{H}: K \times \operatorname{Ann} \mathfrak{h} \rightarrow K \times_{H}$ Ann $\mathfrak{h}$ denote the projection. Then

$\operatorname{Ad}(h) \cdot Z=T \pi_{H} \cdot \nabla_{F_{1}}^{T^{*} K}\left(e, \operatorname{Ad}^{*}(h) \cdot \lambda\right)=\nabla_{F}^{K \times_{H} \operatorname{Ann} \mathfrak{h}}[(h, \lambda)]=T \pi_{H} \cdot \nabla_{F_{1}}^{T^{*} K}(e, \lambda)=Z$ since $F_{1}$ depends only on the second factor, i.e., it is $T^{*} L(K)$-invariant.

Theorem 4.11 (Poisson structure on Weinstein space). The identification

$$
\mathcal{W} / K \stackrel{=}{\longrightarrow} W, \quad[(q ;[q], \eta ; q, \lambda)] \longmapsto([q], \eta ;[(q, \lambda)])
$$

gives an induced Poisson bracket on $C^{\infty}(W)=C^{\infty}(\mathcal{W})^{K}$ which makes the stratified isomorphism

$$
\psi_{0}=\psi_{0}(A): T^{*}(Q / K) \times_{Q / K}\left(\bigsqcup_{q \in Q} \operatorname{Ann}_{q}\right) / K=W \longrightarrow\left(T^{*} Q\right) / K
$$


from Theorem 4.4 into an isomorphism of Poisson spaces.

Let $[(q, \eta, \lambda)] \in \mathcal{W} / K=W$, and $f_{1}, f_{2} \in C^{\infty}(W)$. Assume that $F_{1}, F_{2} \in C^{\infty}(\mathcal{W})^{K}$ are lifts of $f_{1}, f_{2}$ to $\mathcal{W}$. Then the induced Poisson bracket on $W$ is given by

$$
\begin{aligned}
& \left\{f_{1}, f_{2}\right\}^{W}[(q, \eta, \lambda)] \\
& =\Omega_{([q], \eta)}^{Q / K}\left(\left(\left(\Omega_{([q], \eta)}^{Q / K}\right)^{\vee}\right)^{-1}\left(d_{\widetilde{A}} F_{1}(q, \eta, \lambda)\right),\left(\left(\Omega_{([q], \eta)}^{Q / K}\right)^{\vee}\right)^{-1}\left(d_{\widetilde{A}} F_{2}(q, \eta, \lambda)\right)\right) \\
& \quad-\left\langle\lambda, \operatorname{Curv}_{0}^{A}\left(v_{1}(q)^{\operatorname{hor}(\pi)}, v_{2}(q)^{\operatorname{hor}(\pi)}\right)\right\rangle-\left\langle\lambda,\left[d_{v} F_{1}(q, \eta, \lambda), d_{v} F_{2}(q, \eta, \lambda)\right]\right\rangle
\end{aligned}
$$

where $\Omega^{Q / K}$ is the canonical symplectic form on $T^{*}(Q / K)$, the covariant derivatives $d_{\widetilde{A}} F_{i}(q, \eta, \lambda)$ are considered as elements of $T_{([q], \eta)}^{*}\left(T^{*}(Q / K)\right)$, and $v_{i}(q)^{\mathrm{hor}(\pi)}=$ $T \tau \cdot\left(\left(\Omega_{([q], \eta)}^{Q / K}\right)^{\vee}\right)^{-1}\left(d_{\widetilde{A}} F_{i}(q, \eta, \lambda)\right)$. Finally, $\operatorname{Curv}_{0}^{A}$ is the induced form on $Q / K$ associated to the mechanical connection A from Proposition 3.1.

In the case that $K$ acts freely on $Q$ the Poisson bracket on the reduced Poisson manifold $T^{*} Q / K$ is determined in Zaalani [34] and in Perlmutter and Ratiu [23]. In the first paper the realization of $T^{*} Q / K$ as Weinstein space is used, the latter deals with its realization as Sternberg and Weinstein space.

Proof. The first part of the theorem has already been checked in the proof of Theorem 4.4 .

Let $f_{1}, f_{2} \in C^{\infty}(W)$ and let $F_{1}, F_{2} \in C^{\infty}(\mathcal{W})^{K}$ be its unique lifts to $\mathcal{W}$. In order to establish the formula for the reduced Poisson bracket we decompose the Hamiltonian vector fields of $F_{1}$ and $F_{2}$ as above

$$
\nabla_{F_{i}}^{\sigma}(q, \eta, \lambda)=\left(v_{i}(q) ; \eta_{i}^{\prime}([q], \eta) ; v_{i}(q), \nu_{i}(q, \lambda)\right) \quad(i=1,2) .
$$

With the intrinsic symplectic form $\sigma$ on $\mathcal{W}$ from Proposition 3.2 we have

$$
\left\{f_{1}, f_{2}\right\}^{W}[(q, \eta, \lambda)]=\left\{F_{1}, F_{2}\right\}^{\mathcal{W}}(q, \eta, \lambda)=\sigma\left(\nabla_{F_{1}}^{\sigma}, \nabla_{F_{2}}^{\sigma}\right)(q, \eta, \lambda)
$$

which turns to the desired formula by the identity $\eta_{i}^{\prime}([q], \eta)^{\operatorname{hor}(\tau)}=v_{i}(q)^{\operatorname{hor}(\pi)}$, and Lemmas 4.8, 4.9, and 4.10.

The above theorem implies, in particular, that the embedding $T^{*}(Q / K) \hookrightarrow W$ as the zero section is a Poisson morphism when $T^{*}(Q / K)$ is equipped with its standard Poisson structure. Now, fixing $\lambda \in(\operatorname{Ann} \mathfrak{h})_{(L)^{H}}$ defines a smooth embedding

$$
\iota_{\lambda}: T^{*}(Q / K) \cong T^{*}\left(Q_{H} / N(H)\right) \longrightarrow W_{(L)}, \quad([q], \eta) \longmapsto[(q, \eta, \lambda)]
$$

where $q \in Q_{H}:=\left\{q \in Q: K_{q}=H\right\}, N(H)$ is the normalizer of $H$ in $K$, and $(L)^{H}$, $(L)$ denotes the conjugacy class of $L \subseteq H$ in $H, K$ respectively. A quick inspection shows that $\iota_{\lambda}$ is, in general, not Poisson if $T^{*}(Q / K)$ carries its usual Poisson structure. An element $\lambda \in \mathfrak{k}^{*}$ is called totally isotropic if $\mathfrak{k}_{\lambda}=\mathfrak{k}$. 
Corollary 4.12 (Charge). If $\lambda \in A n n \mathfrak{h}$ is totally isotropic then the embedding $\iota_{\lambda}: T^{*}(Q / K) \hookrightarrow W$ is Poisson if $T^{*}(Q / K)$ is equipped with the Poisson structure stemming from the magnetic symplectic form $\Omega^{\lambda}:=\Omega^{Q / K}-\left\langle\lambda, \tau^{*} \operatorname{Curv}_{0}^{A}\right\rangle$.

Proof. This is obvious from the formula of Theorem 4.11.

Due to the appealing similarity of the symplectic form $\Omega^{\lambda}$ with that appearing in electromagnetism $\Omega^{\lambda}$ is called a magnetic symplectic form, and we think of the totally isotropic momentum value $\lambda$ as the charge of the test particles moving in the electromagnetic field on the reduced configuration space $Q / K$. This analogy will be carried further in section 5 where we equip our particles with spin by considering general momentum values. See Guillemin and Sternberg [13, section 20] for a discussion of electromagnetism in a symplectic framework.

Since Hamiltonian vector fields associated to $K$-invariant functions are tangent to the isotropy type submanifolds we can define Hamiltonian vector fields on $W$ as follows. Let $f \in C^{\infty}(W)$ and $F=\phi^{*} f \in C^{\infty}(\mathcal{W})^{K}$ where $\phi: \mathcal{W} \rightarrow W$ is the projection. Then $\nabla_{f}^{W}$ which is characterized by

$$
\nabla_{f}^{W} \circ \phi=-\{f, \cdot\}^{W} \circ \phi=T \phi \cdot \nabla_{F}^{\sigma}
$$

is a stratified vector field on $W$ in the sense of Subsection 2.3. Let $\phi_{(L)}: \mathcal{W}_{(L)} \rightarrow W_{(L)}$ denote the restriction of $\phi$ to the isotropy stratum.

Corollary 4.13 (Hamiltonian vector fields). Let $[(q, \eta, \lambda)] \in W_{(L)}=\mathcal{W}_{(L)} / K$ and $f \in C^{\infty}(W)$ with unique lift $F=\phi^{*} f$. Then

$$
\nabla_{f}^{W}[(q, \eta, \lambda)]=\left(v_{0},\left(\left(\Omega_{([q], \eta)}^{Q / K}\right)^{\vee}\right)^{-1}\left(d_{\widetilde{A}} F(q, \eta, \lambda)\right), \operatorname{ad}^{*}\left(d_{v} F(q, \eta, \lambda)\right) \cdot \lambda\right)
$$

where we consider $d_{\widetilde{A}} F(q, \eta, \lambda)$ as an element of $T_{([q], \eta)}^{*}\left(T^{*}(Q / K)\right)$ through the isomorphism given by the dual of the horizontal lift with respect to the mechanical connection $A$ on $Q \rightarrow Q / K$. Moreover, $\left.v_{0}:=T_{([q], \eta)} \tau \cdot\left(\left(\Omega_{([q], \eta)}^{Q / K}\right)\right)^{\vee}\right)^{-1}\left(d_{\widetilde{A}} F(q, \eta, \lambda)\right)$.

Proof. Notice firstly that

$\nabla_{F}^{\sigma}(q, \eta, \lambda)-\left(v(q)^{\operatorname{hor}(\pi)},\left(\left(\Omega_{([q], \eta)}^{Q / K}\right)^{\vee}\right)^{-1}\left(d_{\widetilde{A}} F(q, \eta, \lambda)\right), \operatorname{ad}^{*}(-Z) \cdot \lambda\right) \in \operatorname{ker} T_{(q, \eta, \lambda)} \phi_{(L)}$

by Lemmas $4.8,4.9$, and 4.10 , and where $-Z=d_{v} F(q, \eta, \lambda)$. We have $v_{0}=T_{q} \pi$. $\left(v(q)^{\operatorname{hor}(\pi)}\right)$ then

$$
\begin{aligned}
\nabla_{f}^{W}[(q, \eta, \lambda)] & =T_{(q, \eta, \lambda)} \phi_{(L)} \cdot \nabla_{F}^{\sigma}(q, \eta, \lambda) \\
& =T_{(q, \eta, \lambda)} \phi_{(L)} \cdot\left(v(q)^{\operatorname{hor}(\pi)},\left(\left(\Omega_{([q], \eta)}^{Q / K}\right)^{\vee}\right)^{-1}\left(d_{\widetilde{A}} F(q, \eta, \lambda)\right), \operatorname{ad}^{*}(-Z) \cdot \lambda\right) \\
& =\left(v_{0},\left(\left(\Omega_{([q], \eta)}^{Q / K}\right)^{\vee}\right)^{-1}\left(d_{\widetilde{A}} F(q, \eta, \lambda)\right), \operatorname{ad}^{*}(-Z) \cdot \lambda\right) .
\end{aligned}
$$


The last equality in the aligned equation is true since

$$
\left(v(q)^{\operatorname{hor}(\pi)},\left(\left(\Omega_{([q], \eta)}^{Q / K}\right)^{\vee}\right)^{-1}\left(d_{\widetilde{A}} F(q, \eta, \lambda)\right), \operatorname{ad}^{*}(-Z) \cdot \lambda\right)
$$

is horizontal with respect to the projection $\phi_{(L)}: \mathcal{W}_{(L)} \rightarrow W_{(L)}$ which is equipped with the structure of a Riemannian submersion.

\section{Symplectic leaves}

It is well known that any smooth Poisson manifold foliates into smooth symplectic initial submanifolds. These symplectic submanifolds are the leaves of the integrable distribution with jumping rank defined by the Hamiltonian vector fields. In particular, if $(M, \omega)$ is a symplectic manifold which is acted upon in a Hamiltonian fashion by a compact Lie group $K$ such that this action is free this is true for the quotient manifold $M / K$ which carries an induced Poisson structure. Moreover, the symplectic leaves of $M / K$ are simply the connected components of the Marsden-Weinstein reduced spaces $M / /{ }_{\mathcal{O}} K$ where $\mathcal{O}$ is a coadjoint orbit.

In the present context where $M / K=\left(T^{*} Q\right) / K$ is a singular Poisson space such a statement cannot be true in this form. (We continue to assume that $Q$ is of single isotropy type, i.e., $Q=Q_{(H)}$ whence the cotangent lifted action has a non-trivial isotropy lattice if $H \neq\{e\}$.) However, using that Hamiltonian vector fields associated to $K$-invariant functions are tangent to isotropy type submanifolds $\left(T^{*} Q\right)_{(L)}$ we can still define a characteristic distribution on $\left(T^{*} Q\right)_{(L)} / K$, and find in subsection 5.3 that its symplectic leaves are the connected components of the smooth symplectic manifold $T^{*}(Q / K) \times_{Q / K}\left(\bigsqcup_{q \in Q} \mathcal{O} \cap A \cap n \mathfrak{k}_{q}\right)_{(L)} / K$. This result is put into perspective in Subsection 5.2 where we recall the singular Weinstein space description of $T^{*} Q / /{ }_{\mathcal{O}} K \cong \mathcal{W} / /{ }_{\mathcal{O}} K$.

\subsection{Universal reduction procedure}

The singular reduction diagram of Ortega and Ratiu [20, Theorem 8.4.4] adjoined to the universal reduction procedure of Arms, Cushman, and Gotay [3], see also [20, section 10.3.2] applied to the Weinstein space has the following form.

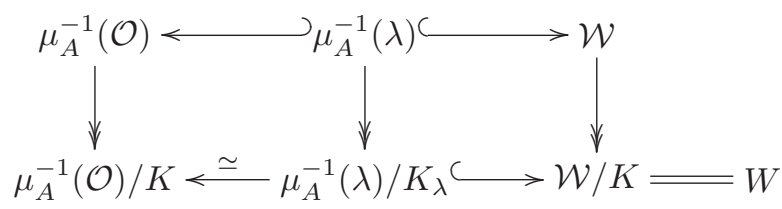

where $\lambda \in \mu_{A}(\mathcal{W})$ and $\mathcal{O}$ is the coadjoint orbit passing through $\lambda$. In this diagram the isomorphism $\mu_{A}^{-1}(\lambda) / K_{\lambda} \cong \mathcal{W} / /{ }_{\mathcal{O}} K$ is an isomorphism of singular symplectic spaces with smooth structure, and the inclusion $\mu_{A}^{-1}(\lambda) / K_{\lambda} \hookrightarrow W$ is a morphism 
of singular Poisson spaces. In the universal reduction scheme of Arms, Cushman, and Gotay [3] this morphism is actually used to equip $\mu_{A}^{-1}(\lambda) / K_{\lambda}$ with a Poisson structure. Therefore, it is sensible to expect the smooth symplectic leaves of $W$ to be the connected components of the smooth symplectic strata of $\mathcal{W} / /{ }_{\mathcal{O}} K$. The latter space is described in the next subsection.

\subsection{Symplectic reduction of $\mathcal{W}$}

Let $\mathcal{O}$ be a coadjoint orbit in the image of the momentum map $\mu_{A}: \mathcal{W} \rightarrow \mathfrak{k}^{*}$, and let $(L)$ be in the isotropy lattice of the $K$-action on $\mathcal{W}$ such that $\mathcal{W}_{(L)}^{\mathcal{O}}:=\mu_{A}^{-1}(\mathcal{O}) \cap \mathcal{W}_{(L)} \neq$ $\emptyset$. Then we have

$$
\iota_{(L)}^{\mathcal{O}}: \mathcal{W}_{(L)}^{\mathcal{O}} \hookrightarrow \mathcal{W},
$$

the canonical embedding, and the orbit projection mapping

$$
\pi_{(L)}^{\mathcal{O}}: \mathcal{W}_{(L)}^{\mathcal{O}} \rightarrow \mathcal{W}_{(L)}^{\mathcal{O}} / K=:\left(\mathcal{W} / /{ }_{\mathcal{O}} K\right)_{(L)} .
$$

Consider furthermore

$$
\rho_{(L)}^{\mathcal{O}}:\left(\bigsqcup_{q \in Q} \mathcal{O} \cap \operatorname{Ann} \mathfrak{k}_{q}\right)_{(L)} \rightarrow\left(\bigsqcup_{q \in Q} \mathcal{O} \cap \operatorname{Ann} \mathfrak{k}_{q}\right)_{(L)} / K
$$

and

$$
\nu_{(L)}^{\mathcal{O}}:\left(\bigsqcup_{q \in Q} \mathcal{O} \cap \operatorname{Ann} \mathfrak{k}_{q}\right)_{(L)} \longrightarrow \mathcal{O}, \quad(q, \lambda) \longmapsto \lambda
$$

as well as the embedding

$$
j_{(L)}^{\mathcal{O}}:\left(\bigsqcup_{q \in Q} \mathcal{O} \cap \operatorname{Ann} \mathfrak{k}_{q}\right)_{(L)} \hookrightarrow \bigsqcup_{q \in Q} \operatorname{Ann} \mathfrak{k}_{q} .
$$

Finally, we denote the Kirillov-Kostant-Souriou symplectic form on $\mathcal{O}$ by $\Omega^{\mathcal{O}}$, that is $\Omega^{\mathcal{O}}(\lambda)\left(\operatorname{ad}^{*}(X) \cdot \lambda, \operatorname{ad}^{*}(Y) \cdot \lambda\right)=\langle\lambda,[X, Y]\rangle$. Remember from Proposition 3.2 that the symplectic structure on $\mathcal{W}$ is denoted by $\sigma$.

Theorem 5.1 (Gauged symplectic reduction). Let $Q=Q_{(H)}$, let $\mathcal{O}$ be a coadjoint orbit in the image of the momentum map $\mu_{A}: \mathcal{W} \rightarrow \mathfrak{k}^{*}$, and let $(L)$ be in the isotropy lattice of the $K$-action on $\mathcal{W}$ such that $\mathcal{W}_{(L)}^{\mathcal{O}}:=\mu_{A}^{-1}(\mathcal{O}) \cap \mathcal{W}_{(L)} \neq \emptyset$. Then the following are true.

(i) The smooth manifolds $\left(\mathcal{W} / /{ }_{\mathcal{O}} K\right)_{(L)}$ and

$$
\left(\mathcal{O} / /_{0} H\right)_{\left(L_{0}\right)^{H}}=:(\mathcal{O} \cap \operatorname{Ann} \mathfrak{h})_{\left(L_{0}\right)^{H}} / H
$$

are typical symplectic strata of the stratified symplectic spaces $\mathcal{W} / /{ }_{\mathcal{O}} K$ and $\mathcal{O} / /{ }_{0} H$ respectively. Here $L_{0}$ is an isotropy subgroup of the induced $H$-action on $\mathcal{O}$ and $\left(L_{0}\right)^{H}$ denotes its isotropy class in $H$. 
(ii) The symplectic stratum $\left(\mathcal{W} / /{ }_{\mathcal{O}} K\right)_{(L)}$ can be globally described as

$$
\left(\mathcal{W} /{ }_{\mathcal{O}} K\right)_{(L)}=T^{*}(Q / K) \times{ }_{Q / K}\left(\bigsqcup_{q \in Q} \mathcal{O} \cap \operatorname{Ann} \mathfrak{k}_{q}\right)_{(L)} / K
$$

whence it is the total space of the smooth symplectic fiber bundle

$$
\left(\mathcal{O} / /{ }_{0} H\right)_{\left(L_{0}\right)^{H}} \complement\left(\mathcal{W} / /{ }_{\mathcal{O}} K\right)_{(L)} \longrightarrow T^{*}(Q / K)
$$

Hereby $L_{0}$ is an isotropy subgroup of the induced $H$-action on $\mathcal{O}$ which is conjugate in $K$ to $L$, and $\left(L_{0}\right)^{H}$ denotes its isotropy class in $H$.

(iii) The symplectic structure $\sigma_{(L)}^{\mathcal{O}}$ on $\left(\mathcal{W} / /{ }_{\mathcal{O}} K\right)_{(L)}$ is uniquely determined and given by the formula

$$
\left(\pi_{(L)}^{\mathcal{O}}\right)^{*} \sigma_{(L)}^{\mathcal{O}}=\left(\iota_{(L)}^{\mathcal{O}}\right)^{*} \sigma-\left(\mu_{A} \mid \mathcal{W}_{(L)}^{\mathcal{O}}\right)^{*} \Omega^{\mathcal{O}}
$$

More precisely,

$$
\sigma_{(L)}^{\mathcal{O}}=\Omega^{Q / K}-\beta_{(L)}^{\mathcal{O}}
$$

where $\beta_{(L)}^{\mathcal{O}} \in \Omega^{2}\left(\left(\bigsqcup_{q \in Q} \mathcal{O} \cap \operatorname{Ann} \mathfrak{k}_{q}\right)_{(L)} / K\right)$ is defined by

$$
\left(\rho_{(L)}^{\mathcal{O}}\right)^{*} \beta_{(L)}^{\mathcal{O}}=\left(j_{(L)}^{\mathcal{O}}\right)^{*} d B+\left(\nu_{(L)}^{\mathcal{O}}\right)^{*} \Omega^{\mathcal{O}} .
$$

Finally $B$ is the form that was introduced in Proposition 3.2. Thus for $(q, \lambda) \in$ $\left(\bigsqcup_{q \in Q} \mathcal{O} \cap \operatorname{Ann} \mathfrak{k}_{q}\right)_{(L)}$ and

$$
\left(v_{i}, \operatorname{ad}^{*}\left(X_{i}\right) . \lambda\right) \in T_{(q, \lambda)}\left(\bigsqcup_{q \in Q} \mathcal{O} \cap \operatorname{Ann} \mathfrak{k}_{q}\right)_{(L)}
$$

where $i=1,2$ we have the explicit formulas

$$
B_{(q, \lambda)}\left(v_{i}, \operatorname{ad}^{*}\left(X_{i}\right) \cdot \lambda\right)=\left\langle\lambda, A_{q}\left(v_{i}\right)\right\rangle
$$

and also

$$
\begin{aligned}
& d B_{(q, \lambda)}\left(\left(v_{1}, \operatorname{ad}^{*}\left(X_{1}\right) \cdot \lambda\right),\left(v_{2}, \operatorname{ad}^{*}\left(X_{2}\right) \cdot \lambda\right)\right) \\
& \quad=\left\langle\lambda, \operatorname{Curv}_{q}^{A}\left(v_{1}, v_{2}\right)\right\rangle+\left\langle\lambda,\left[X_{2}, Z_{1}\right]\right\rangle-\left\langle\lambda,\left[X_{1}, Z_{2}\right]\right\rangle+\left\langle\lambda,\left[Z_{1}, Z_{2}\right]\right\rangle
\end{aligned}
$$

where $v_{i}=\zeta_{Z_{i}}(q) \oplus v_{i}^{\text {hor }} \in \operatorname{Ver}_{q} \oplus \operatorname{Hor}_{q}$ is the decomposition into vertical and horizontal parts with $Z_{i} \in \mathfrak{k}$.

(iv) The stratified symplectic space can be globally described as

$$
\mathcal{W} / /{ }_{\mathcal{O}} K=T^{*}(Q / K) \times{ }_{Q / K} \bigsqcup_{q \in Q} \mathcal{O} \cap \operatorname{Ann} \mathfrak{k}_{q} / K
$$


whence it is canonically the total space of

$$
\mathcal{O} / /{ }_{0} H \longrightarrow \mathcal{W} / /{ }_{\mathcal{O}} K \longrightarrow T^{*}(Q / K)
$$

which is a singular symplectic fiber bundle with singularities confined to the fiber direction in the sense of Definition 2.15.

Proof. Assertion (i) is a well-known principle of stratified symplectic reduction - see Ortega and Ratiu [20, section 8.4]. The other assertions are proved in [14, Theorem 5.5]

\subsection{Symplectic leaves of $W=\mathcal{W} / K$}

Let $\mathcal{O}$ be a coadjoint orbit in the image of the cotangent bundle momentum map $\mu: T^{*} Q \rightarrow \mathfrak{k}^{*}$. As before $(L)$ denotes an isotropy type of the $K$-action on $T^{*} Q$, and $\left(L_{0}\right)^{H}$ denotes an isotropy type of the $\operatorname{Ad}^{*}(H)$-action on $\mathcal{O}$. Let $\lambda \in \mathcal{O} \cap$ Ann $\mathfrak{h}$ with $K_{\lambda} \cap H=L_{0} \subseteq H$. We want to make use of the Witt-Artin decomposition and thus denote the symplectic slice of the $H$-action on $\mathcal{O}$ at $\lambda$ by

$$
V:=(\mathfrak{h} \cdot \lambda)^{\Omega^{\mathcal{O}}} /(\mathfrak{h} \cdot \lambda)
$$

where $\Omega^{\mathcal{O}}$ denotes the positive KKS-form on $\mathcal{O}$. Notice that this is well defined since $H \cdot \lambda=\operatorname{Ad}^{*}(H) \cdot \lambda \subseteq \mathcal{O} \cap \operatorname{Ann} \mathfrak{h}$ is an isotropic submanifold of $\mathcal{O}$. (The $H$-action on $\mathcal{O}$ is Hamiltonian with momentum map $\mathcal{O} \rightarrow \mathfrak{h}^{*}$ given by restriction to $\mathfrak{h}$.) By construction $V$ is a symplectic vector space. Thus this is also true for the linear subspace $V_{L_{0}}:=\left\{v \in V: H_{v}=L_{0}\right\}$ of fixed symmetry type.

Lemma 5.2. Under these assumptions $(\mathcal{O} \cap \operatorname{Ann} \mathfrak{h})_{\left(L_{0}\right)^{H}}$ is a smooth manifold and $T_{\lambda}(\mathcal{O} \cap \operatorname{Ann} \mathfrak{h})_{\left(L_{0}\right)^{H}}=T_{([e], 0)}\left(H / L_{0} \times V_{L_{0}}\right)$. Furthermore, $(\mathcal{O} \cap \operatorname{Ann} \mathfrak{h})_{\left(L_{0}\right)^{H}} / H$ is a smooth symplectic manifold and $T_{[\lambda]}\left((\mathcal{O} \cap \text { Ann } \mathfrak{h})_{\left(L_{0}\right)^{H}} / H\right)=V_{L_{0}}$.

Proof. This is a direct consequence of the Symplectic Slice theorem. See Ortega and Ratiu [20, chapter 7] for a treatment of this theorem and [20, section 8.1] for the way in which it is used.

For notational convenience we abbreviate $(\mathcal{O} \cap \operatorname{Ann} \mathfrak{h})_{\left(L_{0}\right)^{H}} / H=:\left(\mathcal{O} / /{ }_{0} H\right)_{\left(L_{0}\right)^{H}}$.

Lemma 5.3. Assume that $[(q, \eta, \lambda)] \in W_{(L)}=\mathcal{W}_{(L)} / K$ and $f \in C^{\infty}(W)$ with unique lift $F=\phi^{*} f$. Let $q \in Q_{H}$ and $\lambda \in(\text { Ann } \mathfrak{h})_{\left(L_{0}\right)^{H}}$ where $L_{0} \subseteq H$ is conjugate to $L$ within $K$. Then

$$
\nabla_{f}^{W}[(q, \eta, \lambda)] \in T_{([q], \eta)}\left(T^{*}(Q / K)\right) \times T_{[\lambda]}\left(\mathcal{O} /{ }_{0} H\right)_{\left(L_{0}\right)^{H}}
$$

Moreover, the latter space is the real span of local Hamiltonian vector fields evaluated at $[(q, \eta, \lambda)]$. 
Proof. According to Corollary 4.13 we have

$$
\nabla_{f}^{W}[(q, \eta, \lambda)]=\left(v_{0},\left(\left(\Omega_{([q], \eta)}^{Q / K}\right)^{\vee}\right)^{-1}\left(d_{\widetilde{A}} F(q, \eta, \lambda)\right), \operatorname{ad}^{*}\left(d_{v} F(q, \eta, \lambda)\right) \cdot \lambda\right)
$$

where we consider $d_{\widetilde{A}} F(q, \eta, \lambda)$ as an element of $T_{([q], \eta)}^{*}\left(T^{*}(Q / K)\right)$ through the isomorphism given by the dual of the horizontal lift with respect to the mechanical connection $A$ on $Q \rightarrow Q / K$. Moreover, $v_{0}:=T_{([q], \eta)} \tau \cdot\left(\left(\Omega_{([q], \eta)}^{Q / K}\right)^{\vee}\right)^{-1}\left(d_{\widetilde{A}} F(q, \eta, \lambda)\right)$. By Lemma 5.2 we have to check that $\operatorname{ad}^{*}(-Z) . \lambda \in V_{L_{0}}=\left((\mathfrak{h} \cdot \lambda)^{\Omega^{\mathcal{O}}} /(\mathfrak{h} \cdot \lambda)\right)_{L_{0}}$. Indeed, by Lemma 4.10 it is true that $\operatorname{Ad}(h) \cdot Z=Z$ for all $h \in H$ where $Z:=-d_{v} F(q, \eta, \lambda)$. Thus $[Z, \mathfrak{h}]=0$ whence $\operatorname{ad}^{*}(-Z) \cdot \lambda \in(\mathfrak{h} \cdot \lambda)^{\Omega^{\mathcal{O}}}$ and even $\operatorname{ad}^{*}(-Z) \cdot \lambda \in(\mathfrak{h} \cdot \lambda)^{\Omega^{\mathcal{O}}} /(\mathfrak{h} \cdot \lambda)$. Moreover, it follows that $h \cdot \operatorname{ad}^{*}(-Z) \cdot \lambda=\operatorname{ad}^{*}(-Z) \cdot \lambda$ for all $h \in H \cap K_{\lambda}=L_{0}$. However, since $\operatorname{ad}^{*}(-Z) \cdot \lambda$ is also an element of the Riemannian slice of the $H$-action on $\mathcal{O}$ at $\lambda$ it follows that $H_{\mathrm{ad}^{*}(-Z) \cdot \lambda} \subseteq H_{\lambda}=L_{0}$ since this is a general feature of Riemannian slices. Therefore, $\operatorname{ad}^{*}(-Z) \cdot \lambda \in V_{L_{0}}$, as claimed.

The second claim follows by going again through the proof of Lemma 4.10.

Let $P_{(L)}$ denote the coinduced Poisson two-tensor on $W_{(L)}$. Then we may rephrase the above lemma by saying that

$$
\check{P}_{(L)}\left(T_{[(q, \eta, \lambda)]}^{*} W_{(L)}\right)=T_{([q], \eta)}\left(T^{*}(Q / K)\right) \times T_{[\lambda]}\left(\mathcal{O} /{ }_{0} H\right)_{\left(L_{0}\right)^{H}}
$$

Thus we get the following theorem.

Theorem 5.4 (Symplectic leaves). Let $(L)$ be an element of the isotropy lattice of the $K$-action on $\mathcal{W}$. Then the characteristic distribution of the coinduced Poisson structure $P_{(L)}$ on $W_{(L)}$ is given by

$$
\check{P}_{(L)}\left(T^{*} W_{(L)}\right)=T\left(T^{*}(Q / K) \times_{Q / K}\left(\bigsqcup_{q \in Q} \mathcal{O} \cap \operatorname{Ann}^{\mathfrak{k}_{q}}\right)_{(L)} / K\right)
$$

whence the smooth symplectic leaves of $W_{(L)}$ are the connected components of the smooth symplectic manifolds

$$
\left(\mathcal{W} / /_{\mathcal{O}} K\right)_{(L)}=T^{*}(Q / K) \times_{Q / K}\left(\bigsqcup_{q \in Q} \mathcal{O} \cap \operatorname{Ann}_{q}\right)_{(L)} / K .
$$

The symplectic form which makes the inclusion $\left(\mathcal{W} / /_{\mathcal{O}} K\right)_{(L)} \hookrightarrow W_{(L)}$ a Poisson morphism as that of Theorem 5.1.

Proof. The statement about the integrability of the characteristic distribution is tautologous since it is described as the tangent bundle of a smooth manifold. The inclusion $\left(\mathcal{W} /{ }_{\mathcal{O}} K\right)_{(L)} \hookrightarrow W_{(L)}$ is a Poisson morphism by the reasoning of subsection 5.1. 


\subsection{Charge and spin}

We shortly describe and interpret some special cases of Theorem 5.4. Retaining assumptions and notation of this theorem we additionally require that $\left(\bigsqcup_{q \in Q}\right.$ Ann $\left.\mathfrak{k}_{q}\right) / K$ $\rightarrow Q / K$ be a trivial vector bundle so that

$$
\left(\bigsqcup_{q \in Q} \mathcal{O} \cap \operatorname{Ann} \mathfrak{k}_{q}\right)_{(L)} / K=Q / K \times \mathcal{O} / /{ }_{0} H \quad \text { and } \quad \mathcal{W} / /{ }_{\mathcal{O}} K=T^{*}(Q / K) \times \mathcal{O} / /{ }_{0} H .
$$

If $\mathcal{O} / /{ }_{0} H=\{$ point $\}=\{\lambda\}$ then we obtain the same result as in Corollary 4.12, i.e., $T^{*}(Q / K)$ inherits the magnetic symplectic form $\Omega^{\lambda}:=\Omega^{Q / K}-\left\langle\lambda, \tau^{*} \operatorname{Curv}_{0}^{A}\right\rangle$.

If $\mathcal{O} / /{ }_{0} H$ is non-trivial then $\mathcal{W} / /{ }_{\mathcal{O}} K=T^{*}(Q / K) \times \mathcal{O} / /{ }_{0} H$ with its induced symplectic structure

$$
\Omega(q, \eta,[\lambda])=\Omega_{(q, \eta)}^{Q / K}-\left\langle\lambda, \tau^{*} \operatorname{Curv}_{0}^{A}\right\rangle-\Omega_{[\lambda]}^{\mathcal{O}}
$$

(where $\Omega^{\mathcal{O}}$ denotes the reduced symplectic form on $\mathcal{O} / /{ }_{0} H$ obtained from the KKSform on $\mathcal{O}$ ) describes the phase space of a (color)-charged particle moving on $Q / K$ under the influence of the field $\operatorname{Curv}_{0}^{A}$ and with internal spin parameters corresponding to $\mathcal{O} / /{ }_{0} H$.

\section{Examples}

\subsection{Calogero-Moser space}

In the spirit of Hochgerner [14] we can apply Theorem 4.11 to obtain rational versions of spin Calogero-Moser systems. That is, let $V$ be a real Euclidean vector space and $K$ a connected compact Lie group that acts on $V$ through a polar representation. Via the inner product we consider the cotangent bundle of $V$ as a product $T^{*} V=V \times V$. The canonical symplectic form $\Omega$ is thus

$$
\Omega_{(a, \alpha)}\left(\left(a_{1}, \alpha_{1}\right),\left(a_{2}, \alpha_{2}\right)\right)=\left\langle\alpha_{2}, a_{1}\right\rangle-\left\langle\alpha_{1}, a_{2}\right\rangle
$$

where $\langle$,$\rangle is the inner product on V$.

The cotangent lifted action of $K$ is the diagonal action on $V \times V$. According to Dadok [9] we may think of the action by $K$ on $V$ as a symmetric space representation and thus consider $\mathfrak{k} \oplus V=: \mathfrak{l}$ as a real semisimple Lie algebra with Cartan decomposition into $\mathfrak{k}$ and $V$, and with bracket relations $[\mathfrak{k}, \mathfrak{k}] \subseteq \mathfrak{k},[\mathfrak{k}, V] \subseteq V$, and $[V, V] \subseteq V$. The momentum mapping corresponding to the $K$-action on $T^{*} V=V \times V$ is now given by $\mu: V \times V \rightarrow \mathfrak{k}^{*}=\mathfrak{k},(a, \alpha) \mapsto[a, \alpha]=\operatorname{ad}(a) \cdot \alpha$ where we identify $\mathfrak{k}=\mathfrak{k}^{*}$ via an $\operatorname{Ad}(K)$-invariant inner product.

Let $V_{r}$ denote the open and dense subset of $V$ consisting of regular elements. Following the ideas of Alekseevsky, Kriegl, Losik, Michor [1] we can interpret the Poisson reduced space $\left(T^{*} V_{r}\right) / K$ as the Poisson phase space of a rational spin Calogero-Moser system. Indeed, let $H$ denote the free Hamiltonian on $T^{*} V_{r}$ and $h$ its induced Hamiltonian on $\left(T^{*} V_{r}\right) / K$. Then the Hamiltonian vector field of $h$ computed by Theorem 4.11 
and its Corollary 4.13 is exactly minus the one of the Calogero-Moser Hamiltonian in [1, section 6.5]. (The difference in the sign comes from a different convention in defining the Hamiltonian vector field of a function.) According to section 5 the smooth symplectic leaves of $\left(T^{*} V_{r}\right) / K$ are given by the connected components of smooth strata of $\left(T^{*} V_{r}\right) / /{ }_{\mathcal{O}} K$ where $\mathcal{O}$ is a coadjoint orbit in $\mathfrak{k}^{*}$. The latter space is described in $[14$, section 6.3$]$.

\subsection{Orbit type $S O(5) / S O(3)$}

Let $S O(5)$ act on $S^{9} \subseteq \mathbb{R}^{5} \times \mathbb{R}^{5}$ through the diagonal action. We denote by $Q$ the open and dense subset of elements $(v, w) \in S^{9} \subseteq \mathbb{R}^{5} \times \mathbb{R}^{5}$ such that $v$ and $w$ are linearly independent. Clearly, $Q$ is preserved by the $S O(5)$-action, and constitutes, moreover, the regular stratum with respect to this action. Thus $Q$ is of single isotropy type, and this type is easily seen to be $H:=S O(3) \subseteq S O(5)=: K$. Writing $K$ as a matrix group we embed $H$ in the usual way in the lower right corner. The orbit space $Q / K$ can be identified with the open disk $B^{2}$ of radius 1 in $\mathbb{R}^{2}$, and the projection $Q \rightarrow Q / K$ is a (non-principal) fiber bundle with typical fiber $K / H$.

We consider the cotangent lifted $K$-action on $T^{*} Q$. This is clearly a non-free action with a non-trivial isotropy lattice. By Theorem 4.11 the singular Poisson reduced space with respect to the lifted $K$-action is of the form

$$
T^{*} B^{2} \times \operatorname{Ann} \mathfrak{h} / \operatorname{Ad}^{*}(H),
$$

since the bundle $\left(\bigsqcup_{q \in Q}\right.$ Ann $\left.\mathfrak{k}_{q}\right) / K \rightarrow Q / K=B^{2}$ is, in this case, trivial. Using the trace form we identify Ann $\mathfrak{h}$ with $\mathfrak{h}^{\perp}$. Now, the map

$$
\mathfrak{h}^{\perp} \longrightarrow \mathbb{R} \times \mathbb{R}^{3} \times \mathbb{R}^{3}, \quad\left(x_{i j}\right)_{i j} \longmapsto\left(x_{21},\left(x_{k 1}\right)_{k=3}^{5},\left(x_{k 2}\right)_{k=3}^{5}\right)=(t, v, w)
$$

is a linear isomorphism that is equivariant for the $H$-action on the right hand side which acts trivially on the $\mathbb{R}$-factor and in the standard diagonal way on the $\mathbb{R}^{3} \times \mathbb{R}^{3}$ factor. Thus the singular Poisson reduced space with respect to the lifted $K$-action is of the form

$$
T^{*} B^{2} \times \mathbb{R} \times \mathbb{R}^{3} \times_{H} \mathbb{R}^{3} .
$$

However, the induced Poisson structure is not obvious at all (if we did not have Theorem 4.11). The stratification is the product stratification induced by the obvious stratification of $\left(\mathbb{R}^{3} \times \mathbb{R}^{3}\right) / H$. Notice also that the induced form $\operatorname{Curv}_{0}^{A}$ on $B^{2}$ which comes from the mechanical curvature is by Proposition $3.1 \mathbb{R}$-valued and evaluates on the $t$-factor.

In order to get a non-trivial symplectic leaf of $T^{*} B^{2} \times \operatorname{Ann} \mathfrak{h} / \operatorname{Ad}^{*}(H)$ let

$$
\lambda:=\left(\begin{array}{ccccc}
0 & 1 & 1 & 0 & 0 \\
-1 & 0 & -1 & 1 & 0 \\
-1 & 1 & & & \\
0 & -1 & & & \\
0 & 0 & & &
\end{array}\right)
$$


and $\mathcal{O}$ be the (co-)adjoint orbit through $\lambda$. Doing the appropriate linear algebra one sees that $\operatorname{dim} \mathfrak{k}_{\lambda}=2, \operatorname{dim} \mathfrak{k}_{\lambda}^{\perp}=8, \mathfrak{h} \cap \mathfrak{k}_{\lambda}=\{0\}$, and $\operatorname{dim} \mathfrak{h}^{\perp} \cap \mathfrak{k}_{\lambda}^{\perp}=5$. Doing a little more linear algebra the symplectic normal space (see, in particular, subsection 5.3) to the $H$-action on $\mathcal{O}$ at $\lambda$ computes to be

$$
V=\left\{\left(\begin{array}{ccccc}
0 & t & a & 0 & 0 \\
-t & 0 & t & a & 0 \\
-a & -t & & & \\
0 & -a & & & \\
0 & 0 & & &
\end{array}\right): t, a \in \mathbb{R}\right\}
$$

Since $\mathfrak{h} \cap \mathfrak{k}_{\lambda}=\{0\}$ we thus get $V_{L_{0}}=V$ whence $T_{[\lambda]}\left((\mathcal{O} \cap \text { Ann } \mathfrak{h})_{\left(L_{0}\right)^{H}} / H\right)=V$. In particular, the symplectic leaf passing through

$$
T^{*} B^{2} \times\{[\lambda]\} \subseteq T^{*} B^{2} \times \operatorname{Ann} \mathfrak{h} / \operatorname{Ad}^{*}(H)=T^{*} B^{2} \times \mathbb{R} \times \mathbb{R}^{3} \times_{H} \mathbb{R}^{3}
$$

is 6-dimensional. Further and more detailed investigation into this example will be written up elsewhere.

\section{References}

[1] D. V. Alekseevsky, A. Kriegl, M. Losik, and P. W. Michor, The Riemannian geometry of orbit spaces - the metric, geodesics, and integrable systems, Publ. Math. Debrecen 62 (2003), no. 3-4, $247-276$.

[2] D. V. Alekseevsky and P. W. Michor, Differential geometry of $\mathfrak{g}$-manifolds, Differential Geom. Appl. 5 (1995), no. 4, 371-403.

[3] J. M. Arms, R. H. Cushman, and M. J. Gotay, A universal reduction procedure for Hamiltonian group actions, The geometry of Hamiltonian systems (Berkeley, CA, 1989), Math. Sci. Res. Inst. Publ., vol. 22, Springer, New York, 1991, pp. 33-51.

[4] L. Bates and E. Lerman, Proper group actions and symplectic stratified spaces, Pacific J. Math. 181 (1997), no. 2, 201-229.

[5] E. Bierstone, Lifting isotopies from orbit spaces, Topology 14 (1975), no. 3, 245-252.

[6] A. D. Blaom, On geometric and dynamic phases (1998), preprint.

[7] G. E. Bredon, Introduction to compact transformation groups, Academic Press, New York, 1972.

[8] H. Cendra, D. D. Holm, J. E. Marsden, and T. S. Ratiu, Lagrangian reduction, the EulerPoincaré equations, and semidirect products, Geometry of differential equations, Amer. Math. Soc. Transl. Ser. 2, vol. 186, Amer. Math. Soc., Providence, RI, 1998, pp. 1-25.

[9] J. Dadok, Polar coordinates induced by actions of compact Lie groups, Trans. Amer. Math. Soc. 288 (1985), no. 1, 125-137.

[10] M. Davis, Smooth G-manifolds as collections of fiber bundles, Pacific J. Math. 77 (1978), no. 2, $315-363$.

[11] C. Emmrich and H. Römer, Orbifolds as configuration spaces of systems with gauge symmetries, Comm. Math. Phys. 129 (1990), no. 1, 69-94.

[12] M. Goresky and R. MacPherson, Stratified Morse theory, Ergebnisse der Mathematik und ihrer Grenzgebiete (3), vol. 14, Springer-Verlag, Berlin, 1988. 
[13] V. Guillemin and S. Sternberg, Symplectic techniques in physics, Cambridge University Press, Cambridge, 1984.

[14] S. Hochgerner, Singular cotangent bundle reduction and spin Calogero-Moser systems (2004), preprint, available at arXiv:math.SG/0411068.

[15] J. E. Marsden, R. Montgomery, and T. Ratiu, Reduction, symmetry, and phases in mechanics, Mem. Amer. Math. Soc. 88 (1990), no. 436, iv+110.

[16] J. E. Marsden and M. Perlmutter, The orbit bundle picture of cotangent bundle reduction, C. R. Math. Acad. Sci. Soc. R. Can. 22 (2000), no. 2, 35-54.

[17] J. Mather, Notes on topological stability, Harvard, 1970, unpublished.

[18] P. W. Michor, Isometric actions of Lie groups and invariants, Lecture Notes, Univ. Vienna, 1997. available at http://www.mat.univie.ac.at/ michor/tgbook.ps.

[19] J.-P. Ortega and T. S. Ratiu, Singular reduction of Poisson manifolds, Lett. Math. Phys. 46 (1998), no. 4, 359-372.

[20] _ Momentum maps and Hamiltonian reduction, Progress in Mathematics, vol. 222, Birkhäuser Boston Inc., Boston, MA, 2004.

[21] R. S. Palais, On the existence of slices for actions of non-compact Lie groups, Ann. of Math. (2) 73 (1961), 295-323.

[22] R. S. Palais and C.-L. Terng, Critical point theory and submanifold geometry, Lecture Notes in Mathematics, vol. 1353, Springer-Verlag, Berlin, 1988.

[23] M. Perlmutter and T. S. Ratiu, Gauged Poisson structures (2004), preprint.

[24] M. Perlmutter, M. Rodriguez-Olmos, and E. Sousa-Diaz, On the geometry of reduced cotangent bundles at zero momentum (2003), preprint, available at arXiv:math.SG/0310437v1.

[25] M. J. Pflaum, Smooth structures on stratified spaces, Quantization of singular symplectic quotients (Landsman, M. J. Pflaum, and Schlichenmeier, eds.), Progress in Mathematics, vol. 198, Birkhäuser, Basel, 2001, pp. 231-258.

[26] _ Analytic and geometric study of stratified spaces, Lecture Notes in Mathematics, vol. 1768, Springer-Verlag, Berlin, 2001

[27] T. Schmah, A cotangent bundle slice theorem (2004), preprint, available at arXiv:math.SG/ 0409148.

[28] G. W. Schwarz, Smooth functions invariant under the action of a compact Lie group, Topology 14 (1975), 63-68.

[29] R. Sjamaar and E. Lerman, Stratified symplectic spaces and reduction, Ann. of Math. (2) 134 (1991), no. 2, 375-422.

[30] S. Smale, Topology and mechanics. I, Invent. Math. 10 (1970), 305-331.

[31] A. Weinstein, Symplectic V-manifolds, periodic orbits of Hamiltonian systems, and the volume of certain Riemannian manifolds, Comm. Pure Appl. Math. 30 (1977), no. 2, 265-271.

[32] , A universal phase space for particles in Yang-Mills fields, Lett. Math. Phys. 2 $(1977 / 78)$, no. $5,417-420$.

[33] $523-557$.

[34] N. Zaalani, Phase space reduction and Poisson structure, J. Math. Phys. 40 (1999), no. 7, $3431-3438$. 